\begin{tabular}{|c|l|}
\hline Title & Dynamics of traveling pulses in heterogeneous media \\
\hline Author(s) & Nishiura, Y asumasa; Teramoto, Takashi; Y uan, X iaohui; Ueda, Kei-Ichi \\
\hline Citation & $\begin{array}{l}\text { Chaos: An Interdisciplinary Journal of Nonlinear Science, 17(3), 037104 } \\
\text { https://doi.org/10.1063/1.2778553 }\end{array}$ \\
\hline Issue Date & $2007-09$ \\
\hline Doc URL & http://hdl.handle.net/2115/30298 \\
\hline Rights & $\begin{array}{l}\text { Copyright 2007 A merican Institute of Physics. This article may be downloaded for personal use only. A ny other use } \\
\text { requires prior permission of the author and the A merican Institute of Physics. }\end{array}$ \\
\hline Type & article \\
\hline File Information & CHA 17-03.pdf \\
\hline
\end{tabular}

Instructions for use 


\title{
Dynamics of traveling pulses in heterogeneous media
}

\author{
Yasumasa Nishiura \\ Research Institute for Electronic Science, Hokkaido University, Sapporo 060-0812, Japan \\ Takashi Teramoto \\ Chitose Institute of Science and Technology, Chitose 066-8655, Japan \\ Xiaohui Yuan \\ Research Institute for Electronic Science, Hokkaido University, Sapporo 060-0812, Japan \\ Kei-Ichi Ueda \\ Research Institute for Mathematical Sciences, Kyoto University, Kyoto 606-8502, Japan
}

(Received 30 April 2007; accepted 9 August 2007; published online 28 September 2007)

\begin{abstract}
One of the fundamental issues of pulse dynamics in dissipative systems is clarifying how the heterogeneity in the media influences the propagating manner. Heterogeneity is the most important and ubiquitous type of external perturbation. We focus on a class of one-dimensional traveling pulses, the associated parameters of which are close to drift and/or saddle-node bifurcations. The advantage in studying the dynamics in such a class is twofold: First, it gives us a perfect microcosm for the variety of outputs in a general setting when pulses encounter heterogeneities. Second, it allows us to reduce the original partial differential equation dynamics to a tractable finitedimensional system. Such pulses are sensitive when they run into heterogeneities and show rich responses such as annihilation, pinning, splitting, rebound, as well as penetration. The reduced ordinary differential equations (ODEs) explain all these dynamics and the underlying bifurcational structure controlling the transitions among different dynamic regimes. It turns out that there are hidden ordered patterns associated with the critical points of ODEs that play a pivotal role in understanding the responses of the pulse; in fact, the depinning of pulses can be explained in terms of global bifurcations among those critical points. We focus mainly on a bump and periodic types of heterogeneity, however our approach is also applicable to general cases. It should be noted that there appears to be spatio-temporal chaos for a periodic type of heterogeneity when its period becomes comparable with the size of the pulse. () 2007 American Institute of Physics.
\end{abstract}

[DOI: $10.1063 / 1.2778553$ ]

Heterogeneity in the media is the most important and ubiquitous type of external perturbation in dissipative systems. We study how heterogeneity influences the propagating manner of spatially localized traveling waves, especially when they encounter heterogeneities of a bump or periodic type. A variety of outputs emerge through the interaction between the traveling pulse and the heterogeneity-induced-ordered-pattern (HIOP), including pinning, splitting, rebound, and penetration. One of the origins of such rich behaviors is that the pulse has potential instabilities that display a variety of dynamics such as drift, saddle-node, and Hopf bifurcations. A reduction from a partial differential equation (PDE) to a finite-dimensional ordinary differential equation (ODE) is possible near such singularities of codim1 and codim2 types, and the resulting system inherits most of the essential dynamics from the original PDE. It turns out that there are hidden HIOPs associated with the critical points of reduced ODEs. They play a pivotal role in understand the transitions among different responses; in fact, various bifurcations such as Hopf and heteroclinic ones of HIOPs cause the onset of those transitions. For a periodic type of heterogeneity, spatio-temporal chaos is also observed numerically for the motion of a pulse as a result of the interplay between the internal driving force and the potential landscape created by heterogeneity.

\section{INTRODUCTION}

Spatially localized patterns such as pulses and spots are fundamental objects arising in many dissipative systems. ${ }^{1-7}$ One of the recent remarkable discoveries is that there is a class of localized patterns that displays a variety of dynamics such as elastic ball-like behaviors upon collision, selfreplication, self-destruction, and spatio-temporal chaos. ${ }^{8-22}$ This is in sharp contrast with well-known classical excitable waves such as in the FitzHugh-Nagumo (FHN) equations in which annihilation is typically observed upon collision. ${ }^{23}$

One of the origins of such rich behaviors is that the pulses have potential instabilities that display a variety of dynamics when parameters are tuned appropriately. For instance, saddle-node structure causes self-replication ${ }^{24,39}$ or self-destruction, ${ }^{25}$ and drift bifurcation is responsible for the onset of traveling motion. Moreover, a singularity of codim2 type, i.e., a system that has a parameter where two bifurcations occur simultaneously, is known to be responsible for the variety of outputs for the scattering process. ${ }^{14,15,26}$ 
Here we study the dynamics of such pulses when the media is changed from homogeneous to heterogeneous in the form of a bump or periodic manner. A variety of exotic responses emerges through the interaction between the traveling pulse and heterogeneities including rebound, oscillation, pinning, and penetration. Even spatio-temporal chaos is observed for periodic media. Our objective is to elucidate the underlying mechanism causing a variety of dynamics by reducing the PDE dynamics to finite-dimensional ones as well as by employing a global bifurcation viewpoint. Note that the reduced ODE system allows us to treat analytically the slowly varying and rapidly oscillating periodic media (see also Ref. 27 for the front case). The model systems employed here are a three-component reaction diffusion system (1) and the Gray-Scott model (9). In particular, we focus on the case in which one of the kinetic coefficients changes like a bump, as in Fig. 1, with the functional form (2), which turns out to give us fundamental information for periodic media and even more general cases. There is, however, one big difference between these two models: the former has a special type of solutions called defects as explained below near the jump points of heterogeneities; the latter does not. The defect solutions are modified states of the ground state caused by heterogeneities, in other words, they are, loosely speaking, heteroclinic orbits connecting the left ground state to the right one at the jump point. They become homoclinic orbits for the bump case as in Fig. 1(b), since it has two jumps. The ground state is the asymptotic state to which pulses approach as $x \rightarrow \pm \infty$. Such defects have a strong influence over the dynamics when pulses encounter heterogeneities; in fact, pinning occurs when the pulses are trapped by stable defects and depinning occurs due to the global bifurcations such as the heteroclinic one connecting those defects. It is therefore very crucial to know the locations and stabilities of defects in order to clarify the behaviors of pulses in heterogeneous media. In addition to defects, there is another category of a new emerging class of solutions arising even for the defectless case. A typical example is a standing pulse sitting at the center of the bump, as will be discussed in Sec. III, which can be regarded as a survivor of the (unstable) standing pulse solution in homogeneous media after breaking the translation-free symmetry due to the heterogeneity. Such standing pulse solutions also play a pivotal role in understanding pulse dynamics in heterogeneous media. For later convenience, we will refer to these defects and standing pulses as heterogeneity-induced ordered patterns (HIOPs). The issue is to find all relevant HIOPs to pulse dynamics in heterogeneous media and study the interrelation among them as well as their stabilities. We resort to two approaches: one is numerical and the other is analytical.

First, a numerical path-tracking method such as AUTO software $^{28}$ allows us to detect good candidates for HIOPs; in fact, it gives us not only the candidates for HIOPs but also many other kinds of ordered patterns created by heterogeneities, as will be discussed in Sec. II.

Note that the role of HIOP is very close to that of the scattors for the case of head-on collisions of two traveling pulses discussed by Refs. 14 and 15 . This is a reasonable resemblance since the dynamics in heterogeneous media can
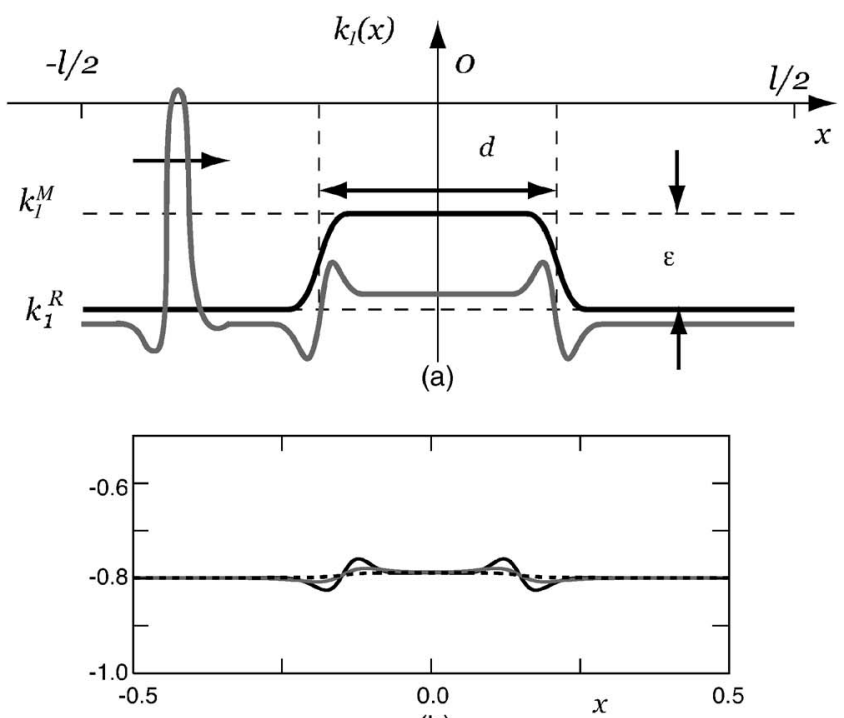

(b)

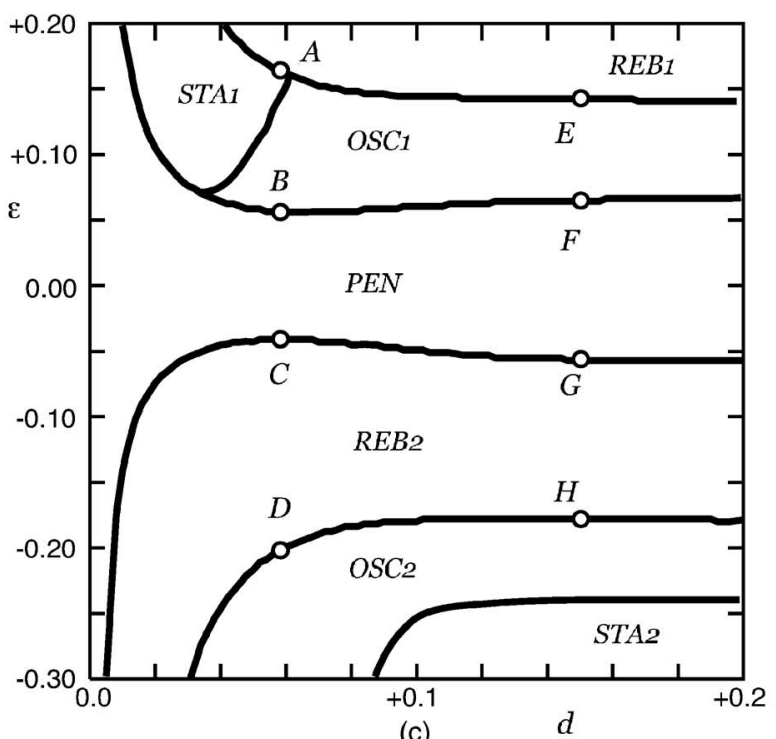

(c)

FIG. 1. (a) Schematic picture of the initial condition for the heterogeneous case. The solid line shows how $k_{1}(x)$ changes in the spatial direction, and the gray line displays the manner in which a traveling pulse hits a small stable defect created by heterogeneity. The profile of a small defect pulse for $(d, \epsilon)=(0.30,0.10)$ is depicted in (b). The solid, gray, and dotted lines indicate the $u, v$, and $w$ components, respectively. (c) The $(d, \boldsymbol{\epsilon})$-phase diagram for the responses of the traveling pulse in the heterogeneous media of bump type. There are seven qualitatively different regimes: penetration (PEN), stationary (STA1, STA2), rebound (REB1, REB2), and oscillatory (OSC1, OSC2). The details of the pattern dynamics observed around the points from A to $\mathrm{H}$ marked by white circles are described in Figs. 2 and 3.

be regarded as a collision process between the traveling pulse and HIOP created by heterogeneities.

Second, we present a powerful reduction method converting PDE dynamics to a finite-dimensional ODE in a similar way to that used in Ref. 29 , which gives us a somewhat normal form for the pulse dynamics in heterogeneous media independent of model systems. The resulting ODEs (see Secs. II and III for details) tell us precisely which ordered patterns detected numerically are relevant to our problem. 
Recall that the pulses treated here are asymptotically stable in homogeneous media, however we are interested in the class of pulses in which the associated parameter values are located close to the singularities, such as drift, saddlenode, and Hopf bifurcations. One of the consequences of this assumption is the enhancement of sensitivity to the perturbations, for instance if there is a traveling pulse close to the drift bifurcation, i.e., the onset of transition from a standing pulse to a traveling one. Its profile is almost symmetric and easy to deform from the right-going pulse to the left-going one by the external perturbations, which actually occur when the pulse encounters the heterogeneity, as will be discussed in Sec. II, and much more exotic dynamics are created than the well-studied case for the FHN equations ${ }^{30-32}$ and the front case. ${ }^{27,33-38}$

The reduced ODEs consist of two parts: one comes from the singularities (hidden instabilities) and the other from heterogeneity (external forces). It should be noted that the abstract form of ODEs remains the same for all types of heterogeneities and the dimension of ODEs depends only on the codimension of the associated singularity. The detailed structure of the heterogeneity is encoded in the convolution integral, i.e., $\Gamma_{i}$ 's of (7), in ODEs. It should be noted that $\Gamma_{i}$ 's also depend on the solution profile and the eigenforms at singularities, which cause the difference in the number of critical points and hence influence their dynamics. It turns out that the ODE dynamics inherits all the PDE dynamics in heterogeneous media, as will be illustrated for the bump case in Sec. II. Quite general results for periodic heterogeneities are obtained for two extreme cases compared with the size of the traveling pulse: short- and long-wave limits. In both cases, the effect coming from the heterogeneity encoded in $\Gamma_{1}$ disappears and the traveling pulses can transmit the media. In the intermediate case, the dynamics becomes more subtle than the above two extreme cases; in fact, spatiotemporal chaos can be observed numerically when the period of the heterogeneity is comparable to the size of the traveling pulse.

Although we only treat the heterogeneities of bump and periodic types, the methods presented here could be applied to more general heterogeneities, which are currently investigated.

When heterogeneity is spatially localized, as with Gaussian distribution, heterogeneity-induced stationary and oscillatory patterns were studied in detail in Refs. 38-40 for the Fitzhugh-Nagumo system and integro-differential equations. The authors found several interesting heterogeneity-induced bifurcations, including the pulse generator, but they did not consider how the traveling pulses interact with those structures.

The paper is organized as follows. In Sec. II, we introduce a three-component model system and the precise form of bump heterogeneity. The phase diagram of PDE dynamics and a reduction method to ODEs are presented. The essential aspects of PDE dynamics are inherited to the ODE dynamics. A detailed study of reduced ODEs clarifies how transitions occur depending on the width and height of the bump. It turns out that HIOPs play a crucial role as separators of those transitions. In Sec. III, we introduce the Gray-Scott model and study the pulse dynamics for bump and periodic media. There appear two HIOPs of standing pulses $S^{ \pm}$(Fig. 20) sitting at the center of the bump and they are connected via an unstable manifold of $S^{+}$. The heteroclinic connection of these two unstable pulses explains three outputs: penetration, splitting, and rebound, when the traveling pulse encounters bump-type heterogeneity. Finally, we present numerically the existence of a spatio-temporal chaos for the periodic media when its period becomes comparable to the size of the pulse. In the Appendix, we derive an ODE system for codim 2 singularity.

\section{BUMP-TYPE HETEROGENEOUS MEDIA WITH DEFECTS}

In order to investigate the dynamics of traveling pulses in heterogeneous media, we employ the following threecomponent reaction-diffusion system, which was proposed as a qualitative model of gas discharge phenomena:

$$
\begin{aligned}
& u_{t}=D_{u} \Delta u+k_{2} u-u^{3}-k_{3} v-k_{4} w+k_{1}, \\
& \tau v_{t}=D_{v} \Delta v+u-v, \quad w_{t}=D_{w} \Delta w+u-w,
\end{aligned}
$$

where $\Delta$ is the Laplacian; $u=u(t, x), v=v(t, x)$, and $w$ $=w(t, x)$ depend on time $t$ and $x \in \mathrm{R}^{1} ; k_{1}, k_{2}, k_{3}$, and $k_{4}$ are kinetic parameters; and $\tau$ and the diffusion coefficients $D_{u}$, $D_{v}$, and $D_{w}$ are positive constants. In view of the nonlinearity, (1) can be regarded as a generalization of the well-known Bonhoeffer-van der Pol kinetics to the three-component system by adding the second inhibitor $w$. The third component $w$ is indispensable for the coexistence of multiple stable traveling spots in higher-dimensional spaces. The system (1) is a simple and prototypical model for the study of interaction among moving particle patterns in dissipative systems. We employ the following parameter values in the subsequent sections: $\quad k_{2}=2.0, \quad k_{3}=1.0, \quad k_{4}=8.5, \quad\left(D_{u}, D_{v}, D_{w}\right)=(0.9$ $\left.\times 10^{-4}, 1.0 \times 10^{-3}, 1.0 \times 10^{-2}\right), \tau=40.0$, and only one parameter $k_{1}$ is varied in the interval $(-7.0,-6.0)$. It was studied in Ref. 41 under this parameter setting that (1) becomes an excitable system with a unique stable equilibrium point and has a stable traveling pulse. The velocity of the pulse is an increasing function of $k_{1}$.

The issue is to study the response of the pulse that encounters the bump-type heterogeneity as shown in Fig. 1(a), especially how it depends on the height $\epsilon$ and width $d$ of the bump. For definiteness, we introduce the following smooth heterogeneity to the parameter $k_{1}$ as $k_{1}(x)=k_{1}^{R}+\epsilon \chi(x, d)$ with even symmetry where

$$
\chi(x, d)=\frac{1}{1+e^{-\gamma(x+d / 2)}}+\frac{1}{1+e^{+\gamma(x-d / 2)}}-1
$$

and $\epsilon \equiv k_{1}^{M}-k_{1}^{R}$ is the height between the middle point and the outer reference domain. From a numerical point of view, we can basically set $k_{1}(-l / 2)=k_{1}(l / 2)=k_{1}^{R}$ and $k_{1}(0)=k_{1}^{M}$ for an appropriate system size $l$ with the Neumann boundary condition. The parameter $\gamma$ controls the steepness of the slope of $k_{1}$ around the jump points $x=-d / 2$ and $+d / 2$. As will be discussed in Sec. III, the dynamics depends also on the slope $\gamma$, however we fix it to be $\gamma=100$ with $l=1.0$ in this 


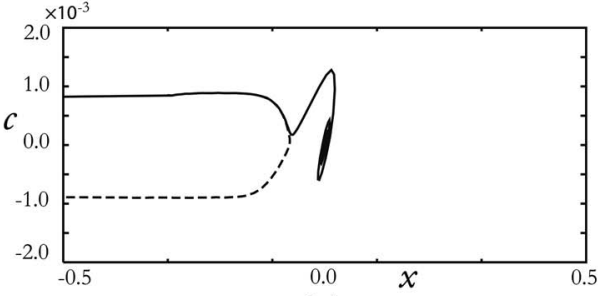

(a)

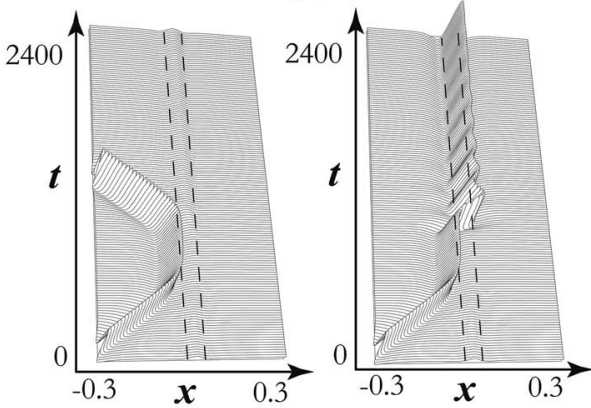

(b)

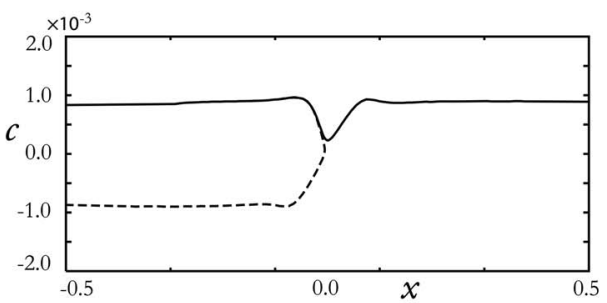

(e)

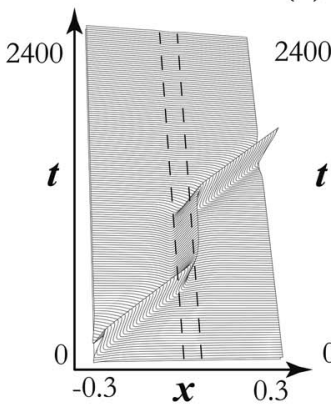

(f)

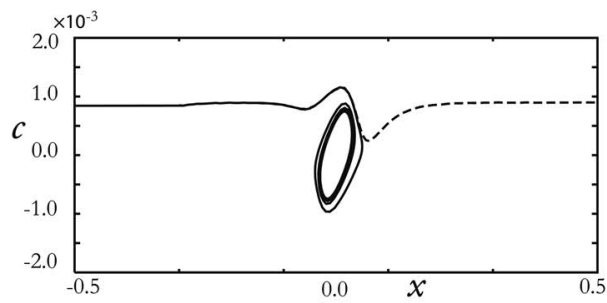

(c)

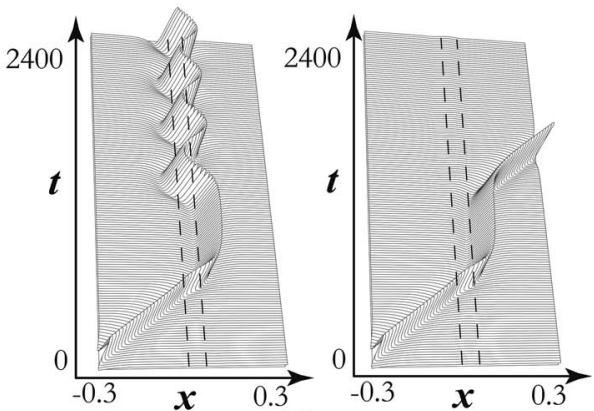

(d)

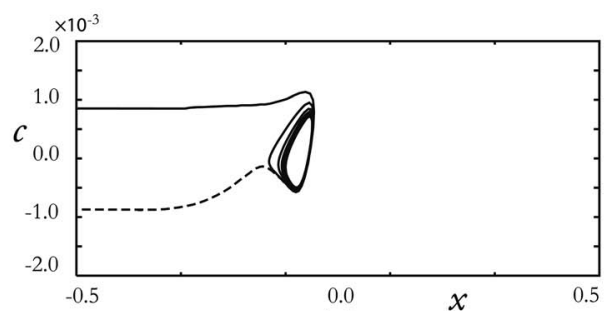

(g)

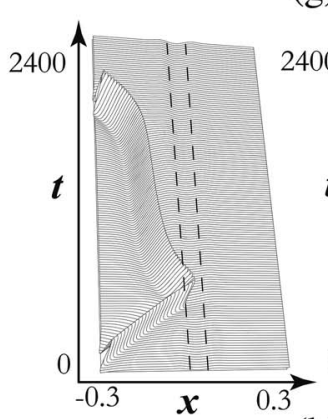

(h)
FIG. 2. The phase portraits and spatio-temporal patterns for the narrow bump case $(\gamma, d)=(100,0.058)$ of the PDE system: (a) and (b) for the transition from REB1 to STA1 around $\epsilon \approx 0.16470$ near point $\mathrm{A}$ in Fig. 1(c); (c) and (d) for the transition from OSC1 to PEN around $\epsilon$ $\approx 0.056849$ near point $\mathrm{B}$; (e) and (f) for the transition from PEN to REB2 around $\epsilon \approx-0.041848$ near point $\mathrm{C}$; and $(\mathrm{g})$ and $(\mathrm{h})$ for the transition from REB2 to OSC2 around $\epsilon \approx$ -0.203173 near point $D$. The horizontal and vertical axes of $(x, c)$ for the portrait denote the peak location and propagating velocity, respectively. section. Note that the heterogeneity (2) satisfies the relation $\chi(x, d)=-\chi(x,-d)$.

Since we introduced the heterogeneity to the kinetic coefficient $k_{1}$ of (1), the uniform state is no longer a background state for the resulting system. There appear many inhomogeneous steady states caused by the $\chi(x, d)$, namely HIOPs (called defects in Ref. 41). We employ the smallest defect in amplitude as the background state for the heterogeneous system, which is shown in Fig. 1(b). Our problem can be reformulated as how the traveling pulse responds when it encounters the background defect. In other words, it is a collision problem between the pulse and defect as schematically shown in Fig. 1(a). The parameter value $k_{1}$ in the reference domain (i.e., far from the bump) is taken to be $k_{1}^{R}$ $=k^{c}+\eta$ with $k_{1}^{c}=-6.79$ and $\eta=+0.29$, in which $k_{1}^{c}$ is a drift bifurcation point from the standing pulse of (1) (see Ref. 41 for details). The reduction from PDE to ODE is valid for small $\eta$. Note that the phase diagram Fig. 1(c) of pulse response does not change qualitatively even for smaller $\eta$.

\section{A. Phase diagram of PDE dynamics}

The outputs of collisions are classified as in the phase diagram of Fig. 1(c), depending on two parameters $(d, \epsilon)$. The pulse is heading to the good (bad) environment for $\epsilon>(<) 0$ in the sense that traveling velocity is larger (smaller) inside of the bump. There are seven different outputs: penetration (PEN), rebound (REB1, REB2), stationary (STA1, STA2), and oscillatory (OSC1, OSC2) states depending on $(d, \epsilon)$. The typical spatio-temporal patterns and their phase portraits are shown in Figs. 2 and 3.

Although it looks trivial that penetration occurs when $|\epsilon|$ is small, i.e., pulses can go across the low barriers, the 


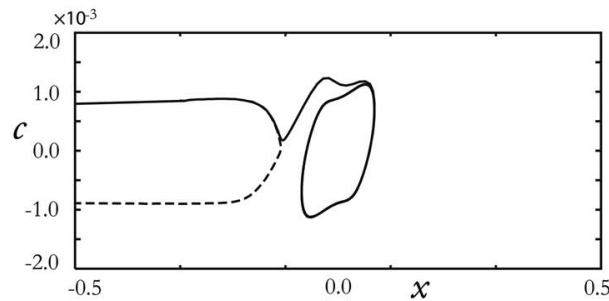

(a)

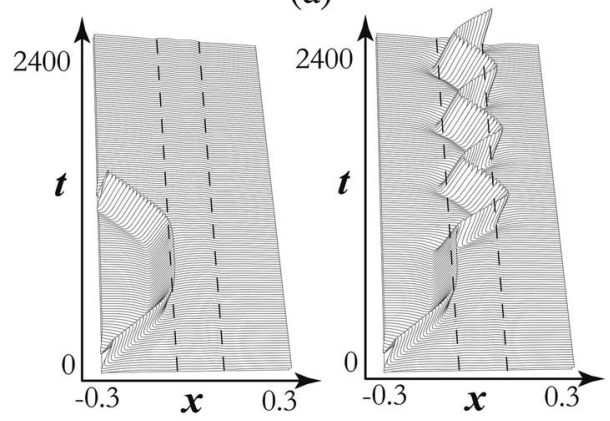

(b)

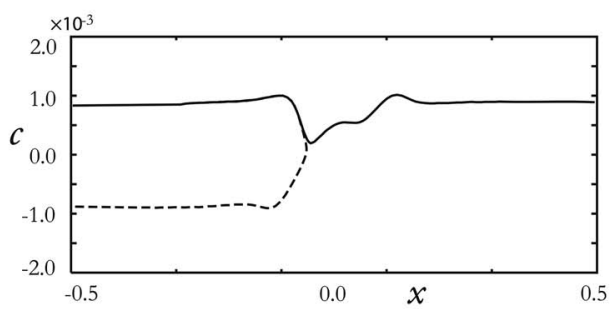

(e)

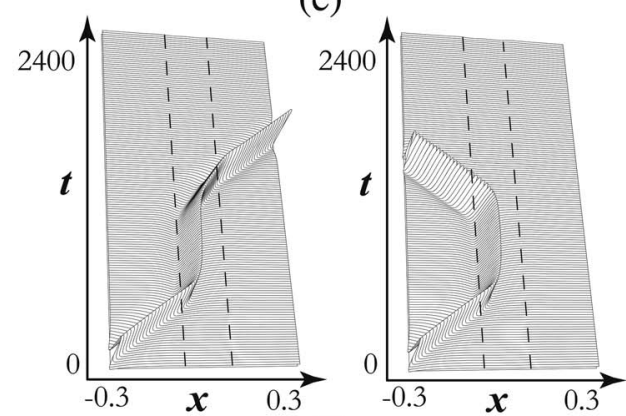

(f)

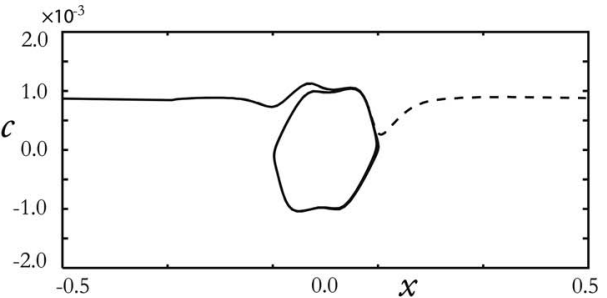

(c)

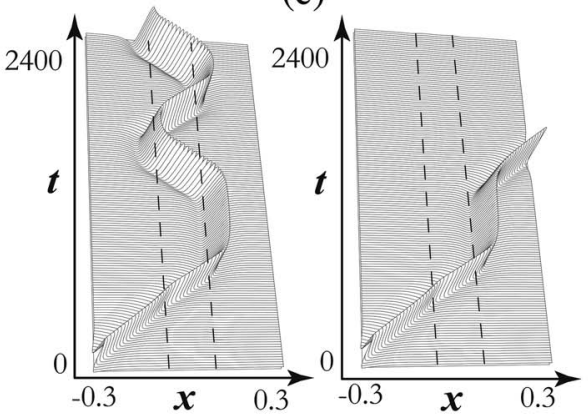

(d)

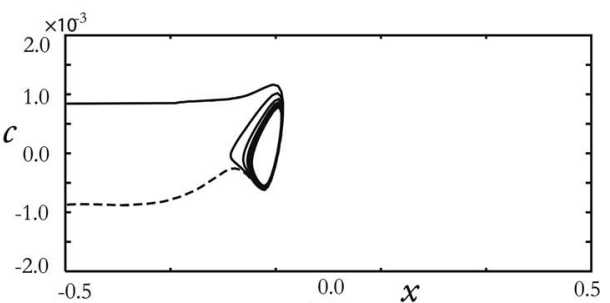

(g)
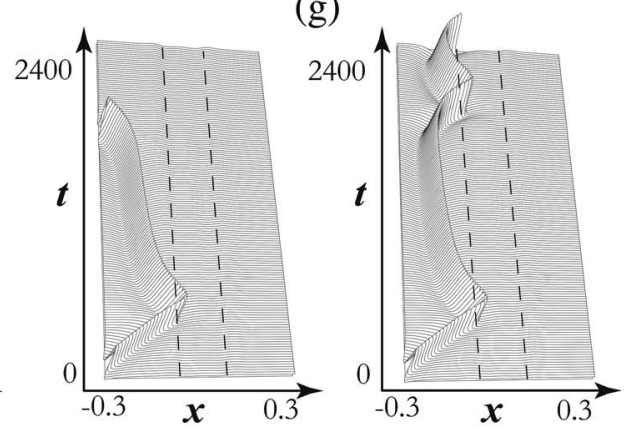

(h)

FIG. 3. The phase portraits and spatio-temporal patterns for the wide bump case $(\gamma, d)=(100,0.15)$ of the PDE system: (a) and (b) for the transition from REB1 to OSC1 around $\epsilon \approx 0.142809$ near point $\mathrm{E}$ in Fig. 1(c); (c) and (d) for the transition from OSC1 to PEN around $\epsilon$ $\approx 0.065405$ near point $\mathrm{F}$; (e) and (f) for the transition from PEN to REB2 around $\epsilon \approx-0.056445$ near point $\mathrm{G}$; and $(\mathrm{g})$ and $(\mathrm{h})$ for the transition from REB2 to OSC2 around $\epsilon \approx$ -0.178858 near point $\mathrm{H}$. The horizontal and vertical axes of $(x, c)$ for the portrait denote the peak location and propagating velocity, respectively. threshold value of the height depends on the velocity (or the distance to the drift bifurcation point). In fact, the penetration regime shrinks when $\eta$ becomes smaller.

As $|\epsilon|$ is increased, the behavior depends on the sign of $\epsilon$. The pulse is trapped by the standing pulse at the center of the bump [Fig. 2(b)] or the oscillatory state [Figs. 2(d), 3(b), and $3(\mathrm{~d})]$ for positive $\epsilon$. On the other hand, the pulse rebounds for negative $\epsilon$. For larger $\epsilon$, rebound occurs for the positive case and trapping to an oscillatory or a stationary state is observed for the negative case.

Let us look carefully at the behaviors of the pulse near the transition points from $\mathrm{A}$ to $\mathrm{H}$ in Fig. 1(c). In view of the numerics of Figs. 2 and 3, it is seen that a common feature for all transitions except D and $\mathrm{H}$ (i.e., REB2-OSC2 transition) is that the solution takes a quasistatic form before it makes a final decision to go. The location of each quasistatic solution is changed depending on $\epsilon$. For instance, it is slightly outside of the left edge of the bump for REB1-STA1 and REB1-OSC1 transitions [see Figs. 2(b) and 3(b)], but it changes to the inside of the left edge for OSC1-PEN transition [see Figs. 2(f) and 3(f)]. For PEN-REB2 transition, its location is changed to the middle of the bump. Those quasistatic states turn out to be HIOPs in later subsections. Note that the two transitions STA1-OSC1 and OSC2-STA2 can be understood by the Hopf bifurcation for the standing pulse located in the middle of the bump, as will be shown later. The excluded case of transition REB2-OSC2 is subtle. In fact, more detailed asymptotic analysis of the oscillatory solution in the OSC2 regime is necessary to understand the transition to rebound, which will be discussed in the next subsection. In the following subsections, we will discuss the underlying mechanism responsible for those transitions. We introduce a reduction method from PDE to finitedimensional dynamics (a set of ODEs), which tells us explicitly the existence and stability of equilibria and time-periodic solutions. The resulting finite-dimensional system is valid 

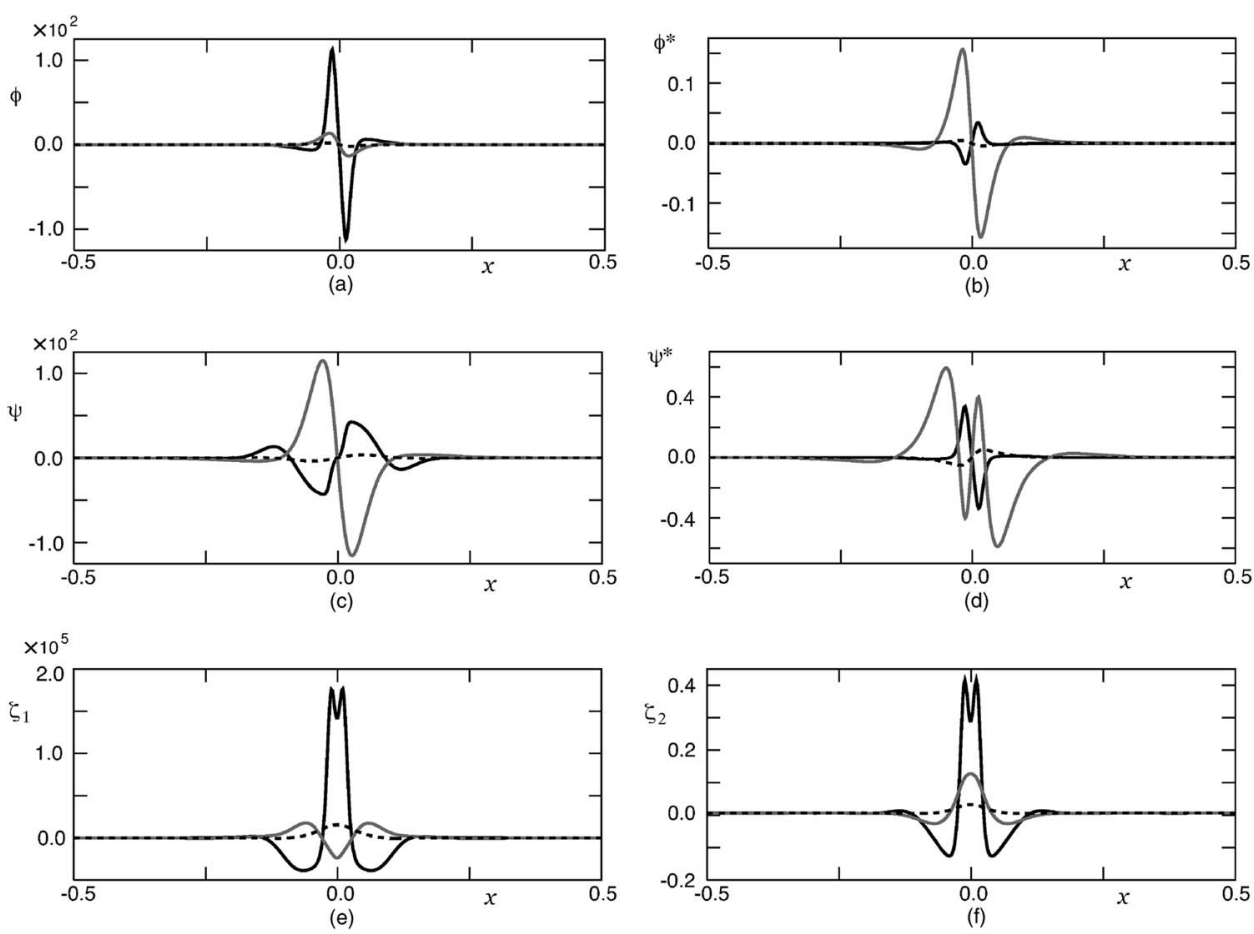

FIG. 4. The profiles of the associated eigenfunctions and their adjoints of the linearized operator at the drift bifurcation point of $\left(k_{1}^{c}=-6.79, \tau\right.$ $=40.0$ ) are depicted in (a) $\phi$, (b) $\phi^{*}$, (c) $\psi$, (d) $\psi^{*}$, (e) $\zeta_{1}$, and (f) $\zeta_{2}$. The solid, gray, and dotted lines correspond to the $u, v$, and $w$ components, respectively. when the parameter is near singularity (i.e., drift bifurcation) and has a universal form independent of specific model systems. The drift bifurcation is the one from the standing pulse to the traveling one and is of pitchfork type.

\section{B. Reduction to ODEs with drift instability}

The pulse dynamics in heterogeneous media can be reduced to a finite-dimensional one when the associated parameter values are close to the drift bifurcation, namely the pulse velocity is slow. It is numerically confirmed that no new types of dynamics appear even if the pulse velocity and the height of the bump become larger, which suggests that the restriction to the neighborhood of the drift instability does not become an essential barrier for understanding the general case. The traveling pulse may have other types of potential instabilities such as saddle-node and Hopf bifurcations. In principle, the reduction is possible for such a higher codimension case as shown in the Appendix. Here we present the reduction method under a general setting for the codim 1 case with drift instability. The codim 2 case is delegated to Sec. III.

Let us present a reduction method from PDE dynamics to a finite-dimensional one in heterogeneous media for the following general $1 \mathrm{D}$ reaction-diffusion system:

$$
\boldsymbol{u}_{t}=D \boldsymbol{u}_{x x}+F(\boldsymbol{u} ; k)=: \mathcal{A}(\boldsymbol{u} ; k), \quad t>0, x \in \mathbb{R},
$$

in which parameter $k \in \mathbb{R}^{1}$ is close to the drift bifurcation point [see paragraph (S3)]. Let $X:=\left\{L^{2}(\mathbb{R})\right\}^{N}, \boldsymbol{u}=\boldsymbol{u}(t, x)$ $=\left(u_{1}, \ldots, u_{N}\right)^{T} \in X$ be an $N$-dimensional vector, $D$ a positive diagonal matrix, $F: \mathbb{R}^{N} \rightarrow \mathbb{R}^{N}$ a smooth vector-valued function, and $\chi(x)$ a $C^{1}$ function. We assume the following for Eq. (3):

(S1) There exists a $k=k^{c}$ such that the nontrivial standing pulse solution $S(x ; k)$ of Eq. (3) exists, i.e., $\mathcal{A}\left(S ; k^{c}\right)=0$.
We consider Eq. (3) in a neighborhood of $k=k^{c}$, with small parameter $\eta$ as $k=k^{c}+\eta$. In order to avoid the unnecessary complexities, we assume that Eq. (3) takes the following form:

$$
\boldsymbol{u}_{t}=\mathcal{A}\left(\boldsymbol{u} ; k^{c}\right)+[\eta+\boldsymbol{\epsilon} \chi(x)] \boldsymbol{g}(\boldsymbol{u}),
$$

where $\boldsymbol{g}$ is an $N$-dimensional vector-valued function. For example, $\boldsymbol{g}={ }^{t}(1,0,0)$ for the model (1).

Let $L$ be the linearized operator of $\mathcal{A}$ with respect to $\boldsymbol{u}$ at $\boldsymbol{u}=S(x, k)$ at $k=k^{c}$, i.e., $L=\mathcal{A}^{\prime}\left(S(x) ; k^{c}\right)$, where "," stands for the Fréchet derivative with respect to $\boldsymbol{u}$, and $L^{*}$ for the adjoint operator of $L$ with respect to $\left\{L^{2}(\mathbb{R})\right\}^{N}$.

(S2) The spectral set of $L$ consists of two sets $\sigma_{1}=\{0\}$ and $\sigma_{2} \subset\left\{\mu \in \mathbb{C} ; \operatorname{Re}(\mu)<-\gamma_{0}\right\}$, where $\gamma_{0}$ is a positive constant.

(S3) $L$ has a singularity at $k=k^{c}$ consisting of drift bifurcation in addition to the translation-free 0 eigenvalue. That is, there exist two eigenfunctions $\phi(x)$ and $\psi(x)$ such that

$$
L \phi=0, \quad L \psi=-\phi,
$$

where $\phi=\partial S / \partial x$. Note that $\phi(x)$ and $\psi(x)$ are odd functions. $\psi(x)$ represents the deformation vector with Jordan form for the drift bifurcation.

Similar properties also hold for $L^{*}$. That is, there exist $\phi^{*}$ and $\psi^{*}$ such that $L^{*} \phi^{*}=0$ and $L^{*} \psi^{*}=-\phi^{*}$, where $\phi^{*}(x)$ and $\psi^{*}(x)$ are odd functions.

(S4) Each element of eigenfunctions $\phi, \phi^{*}, \psi$, and $\psi^{*}$ decays exponentially as $|x| \rightarrow+\infty$.

The above assumptions are numerically confirmed for Eq. (1), in which the kinetic parameter $k_{1}$ is varied. In fact, Fig. 4 shows the profiles of associated adjoint eigenfunctions at the drift bifurcation point $k_{1}^{c}=-6.79$. See Refs. 41 and 42 for details.

Remark 1. Under the normalization of 


$$
\langle\psi, \phi\rangle=\left\langle\psi, \psi^{*}\right\rangle=0, \quad\left\langle\phi, \psi^{*}\right\rangle=1,
$$

all the eigenfunctions are uniquely determined (see, e.g., Ref. 10). We note that

$$
\left\langle\phi, \phi^{*}\right\rangle=0, \quad\left\langle\psi, \phi^{*}\right\rangle=1
$$

hold automatically.

Here $\langle\cdot, \cdot\rangle$ stands for an $L^{2}$ inner product. Let $E$ : $=\operatorname{span}\{\phi, \psi\}$.

Our ansatz is the following:

$$
\boldsymbol{u}=S(x-p)+q \psi(x-p)+\zeta^{\dagger}(x-p)+\boldsymbol{w},
$$

where $p$ and $q$ are scalar functions of time $t ; p$ denotes the location of the pulse, and $q$ denotes its velocity. The remaining two terms $\zeta^{\dagger}$ and $w$ belong to $E^{\perp}$. More precisely, $\zeta^{\dagger}$ $=q^{2} \zeta_{1}+\eta \zeta_{2}$ with $\zeta_{j} \in E^{\perp}$ being determined by

$$
L \zeta_{1}+\frac{1}{2} F^{\prime \prime}(S) \psi^{2}+\psi_{x}=0, \quad L \zeta_{2}+g(S)=0 .
$$

Let $\mathcal{L}(X ; X)$ be all bounded bilinear operators from $X$ to $X$. Since $\mathcal{L}(X, \mathcal{L}(X ; X))$ is identified with $\mathcal{L}(X \times X ; X)$, we represent $\quad\left[F^{\prime}(S) \boldsymbol{u}\right]^{\prime} \boldsymbol{v} \in \mathcal{L}(X ; \mathcal{L}(X ; X)) \quad(\boldsymbol{u}, \boldsymbol{v} \in X) \quad$ as $F^{\prime \prime}(S) \boldsymbol{u} \cdot \boldsymbol{v} \in \mathcal{L}(X \times X ; X)$, and we write $F^{\prime \prime}(S) \boldsymbol{u} \cdot \boldsymbol{u}$ as $F^{\prime \prime}(S) \boldsymbol{u}^{2}$ for simplicity. The third-order derivatives $F^{\prime \prime \prime}(S)$ are similarly defined.

Let $X^{\alpha}$ be the fractional power of space $X$ with respect to the linearized operator $L$ with $\alpha \in(3 / 4,1)$, and $\|\cdot\|_{\alpha}$ denotes the norm of $X^{\alpha}$. Then the remainder term $w \in E^{\perp}$ satisfies the estimate $\|\boldsymbol{w}\|_{\alpha}=O\left(|q|^{3}+|\eta|^{3 / 2}\right)$.

Substituting Eq. (4) into Eq. (3), we obtain

$$
\begin{aligned}
-\dot{p}( & \left.\phi+q \psi_{x}+\zeta_{x}^{\dagger}+\boldsymbol{w}_{x}\right)+\dot{q}\left(\psi+\zeta_{q}^{\dagger}\right)+\boldsymbol{w}_{t} \\
= & L \boldsymbol{w}-q S_{x}+L \zeta^{\dagger}+\frac{1}{2} q^{2} F^{\prime \prime}(S) \psi^{2}+q F^{\prime \prime}(S) \psi \cdot \zeta^{\dagger} \\
& +\frac{1}{6} q^{3} F^{\prime \prime \prime}(S) \psi^{3}+\epsilon \chi(x) \boldsymbol{g}(S)+\eta \boldsymbol{g}(S) \\
& +q\left[\epsilon \chi(x) \boldsymbol{g}^{\prime}(S)+\eta \boldsymbol{g}^{\prime}(S)\right] \psi+\frac{1}{2} \eta^{2} F^{\prime \prime}(S) \zeta_{2}^{2} \\
& +O\left[(|q|+|\eta|)\left(|q|^{3}+|\eta|^{3 / 2}\right)+|\epsilon|(|q|+|\eta|+|\epsilon|)\right] .
\end{aligned}
$$

Taking the inner product of Eq. (5) and $\psi^{*}(x-p)$, we have

$$
\begin{aligned}
\dot{p}= & q-\epsilon\left\langle\chi(x) \boldsymbol{g}(S), \psi^{*}(x)\right\rangle \\
& +O\left[|q|^{3}+|\eta|^{3 / 2}+|\epsilon|(|q|+|\eta|+|\epsilon|)\right] .
\end{aligned}
$$

Similarly, by taking the inner product of Eq. (5) with $\phi^{*}(x-p)$, we obtain the following equation:

$$
\begin{aligned}
\dot{q}= & q^{3}\left(\left\langle F^{\prime \prime}(S) \psi \cdot \zeta_{1}, \phi^{*}\right\rangle+\frac{1}{6}\left\langle F^{\prime \prime \prime}(S) \psi^{3}, \boldsymbol{\phi}^{*}\right\rangle\right. \\
& \left.+\left\langle\partial_{x} \zeta_{1}, \phi^{*}\right\rangle\right)+\epsilon\left\langle\chi(x) \boldsymbol{g}(S), \boldsymbol{\phi}^{*}\right\rangle \\
& +q \eta\left[\left\langle\partial_{x} \zeta_{2}, \boldsymbol{\phi}^{*}\right\rangle+\left\langle\boldsymbol{g}^{\prime}(S) \psi, \boldsymbol{\phi}^{*}\right\rangle+\left\langle F^{\prime \prime}(S) \psi \cdot \zeta_{2}, \boldsymbol{\phi}^{*}\right\rangle\right] \\
& +O\left[(|q|+|\eta|)\left(|q|^{3}+|\eta|^{3 / 2}\right)+|\epsilon|(|q|+|\eta|+|\epsilon|)\right] .
\end{aligned}
$$

The resulting ODEs for $(p, q)$ are given by

$$
\begin{aligned}
\dot{p}= & q-\epsilon \Gamma_{0}(p) \\
& +O\left[|q|^{3}+|\eta|^{3 / 2}+|\epsilon|(|q|+|\eta|+|\epsilon|)\right],
\end{aligned}
$$
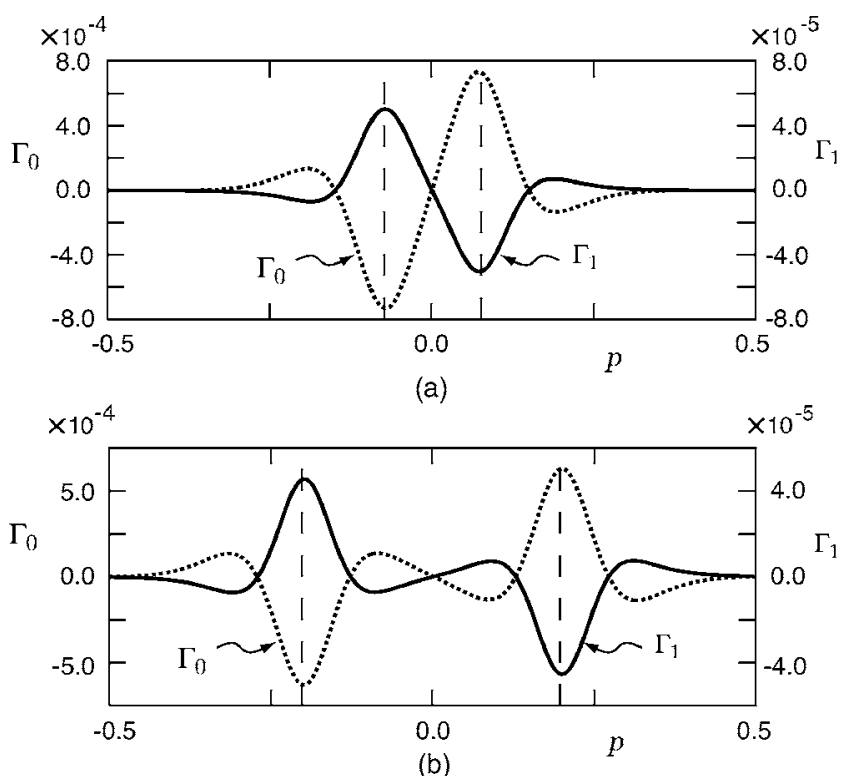

FIG. 5. (a) The profiles of the heterogeneity functions $\Gamma_{i}$ (a) for the narrow bump of $(\gamma, d)=(30,0.15)$ and (b) for the wide bump of $(\gamma, d)=(30,0.40)$. The dotted and solid lines correspond to $\Gamma_{0}$ and $\Gamma_{1}$, respectively.

$$
\begin{aligned}
\dot{q}= & M_{1} q^{3}+M_{2} q \eta+\epsilon \Gamma_{1}(p) \\
& +O\left[(|q|+|\eta|)\left(|q|^{3}+|\eta|^{3 / 2}\right)+|\epsilon|(|q|+|\eta|+|\epsilon|)\right],
\end{aligned}
$$

where

$$
\begin{aligned}
& M_{1}=\left\langle F^{\prime \prime}(S) \psi \cdot \zeta_{1}, \phi^{*}\right\rangle+\frac{1}{6}\left\langle F^{\prime \prime \prime}(S) \psi^{3}, \phi^{*}\right\rangle+\left\langle\partial_{x} \zeta_{1}, \phi^{*}\right\rangle, \\
& M_{2}=\left\langle F^{\prime \prime}(S) \psi \cdot \zeta_{2}, \phi^{*}\right\rangle+\left\langle\partial_{x} \zeta_{2}, \phi^{*}\right\rangle+\left\langle\boldsymbol{g}^{\prime}(S) \psi, \phi^{*}\right\rangle,
\end{aligned}
$$

and the effect of the heterogeneity is represented by the following integral forms:

$$
\begin{aligned}
& \Gamma_{0}(p)=\int_{-\infty}^{\infty} \chi(x) g[S(x-p)] \cdot \psi^{*}(x-p) d x, \\
& \Gamma_{1}(p)=\int_{-\infty}^{\infty} \chi(x) g[S(x-p)] \cdot \phi^{*}(x-p) d x .
\end{aligned}
$$

The principal part is given by the following system:

$$
\dot{p}=q-\epsilon \Gamma_{0}(p), \quad \dot{q}=M_{1} q^{3}+M_{2} q \eta+\epsilon \Gamma_{1}(p) .
$$

Here $p$ stands for the location of the pulse, $q$ for its velocity, and hence the effect of heterogeneity becomes acceleration (deceleration) when $\epsilon \Gamma_{1}>(<) 0$.

The coefficients $M_{1}, M_{2}$ and heterogeneous terms $\Gamma_{i}(p)$ depend on the model system and have a great deal of influence over the dynamics. For our system (1), by using the eigenforms in Fig. 4, they are computed as $M_{1}=-11451$ $<0, M_{2}=0.031>0$, and profiles of $\Gamma_{i}$ are obtained numerically as in Fig. 5. Note that the number of zero points of $\Gamma_{i}$ is increased as the width of the bump is increased, which causes the change of dynamics, as will be discussed in the next subsection. In contrast, the heterogeneous terms become simpler for the Gray-Scott model in Sec. III and the number of zeros does not depend on the width, nevertheless it has a rich dynamics. For the homogeneous case of $\epsilon=0$, the dy- 


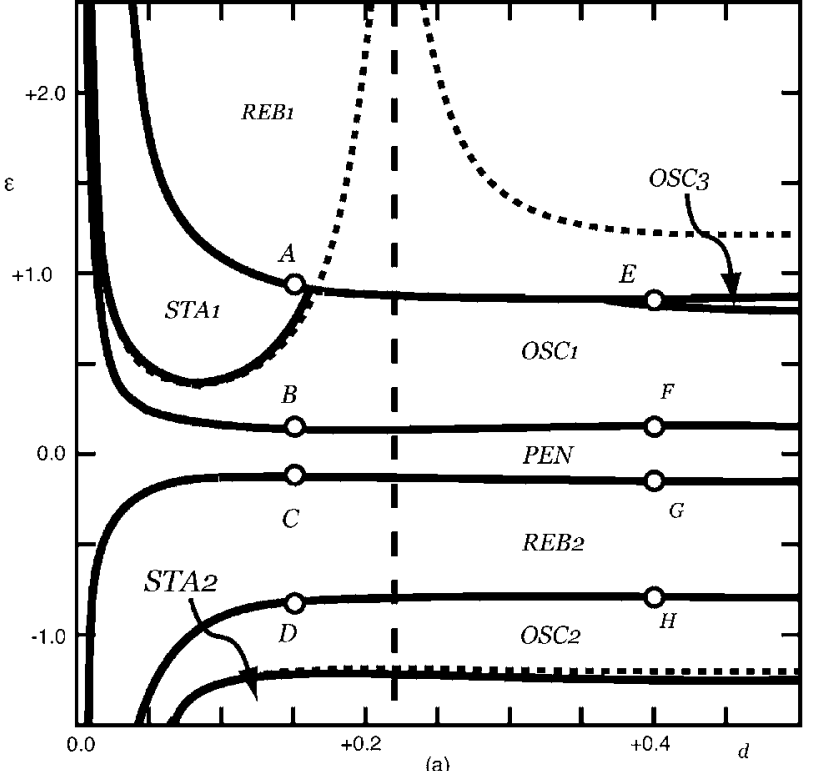

(a)
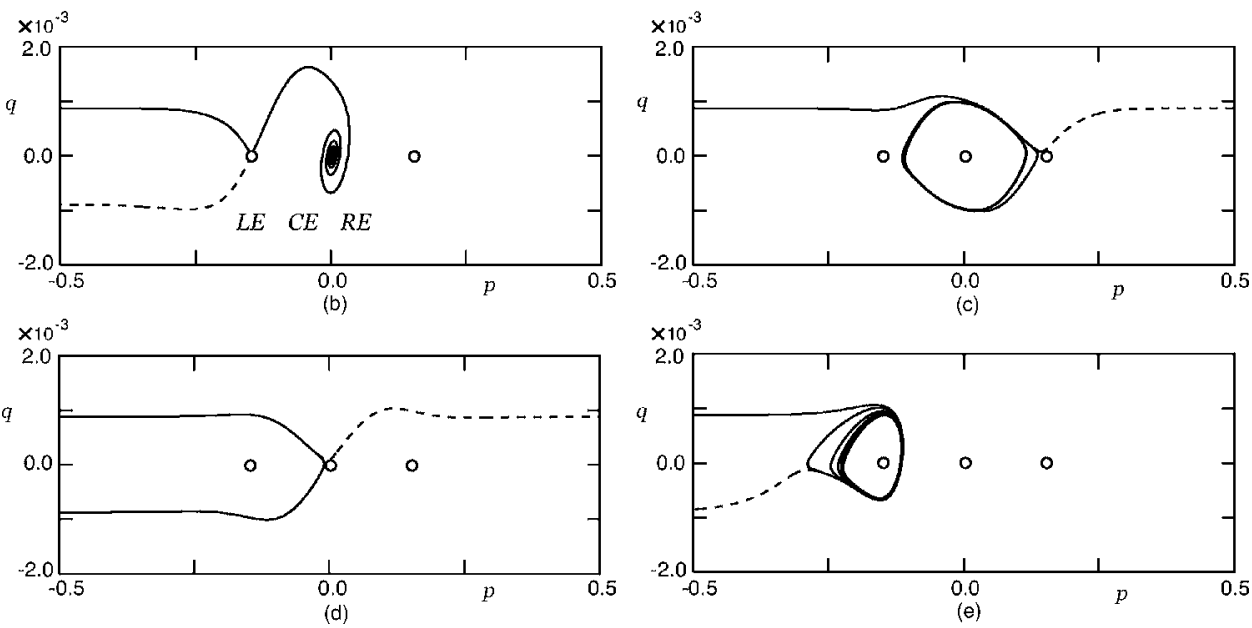

FIG. 6. (a) Phase diagram of ODE dynamics (8) for $\gamma=30$. There are eight types of behaviors whose phase boundaries are indicated by solid lines. The dotted lines corresponds to the Hopf bifurcation lines for the equilibrium points. By increasing the bump width $d$, the pitchfork bifurcation for the center equilibrium point CE occurs along the broken line. Orbit flows in $(p, q)$ space for the narrow bump case of $(\gamma, d)=(30,0.15)$ are depicted in (b) for the transition from REB1 to STA1 around $\epsilon$ $\approx 0.916$ near point $\mathrm{A}$ in (a), (c) for the transition from OSC1 to PEN around $\epsilon \approx 0.143$ near point $\mathrm{B}$, (d) for the transition from PEN to REB2 around $\epsilon \approx-0.136$ near point $\mathrm{C}$, and (e) for the transition from REB2 to OSC2 around $\epsilon \approx-0.823$ near point D. The solid and open circles indicate the stable and unstable stationary states, respectively. namics is reduced to study a single equation of second-order independent of $p$. The equilibrium point $E_{1}$ of $q=0$ corresponds to the standing pulse solutions, and $E_{2}^{ \pm}$given by $q$ $= \pm \sqrt{-M_{2} \eta / M_{1}}$ for $\eta>0$ correspond to the right- and leftgoing traveling pulses. As $\eta$ is changed from negative to positive, the drift bifurcation occurs and $E_{2}^{ \pm}$of $q \neq 0$ bifurcate from $E_{1}$ supercritically. Since we are interested in the regime in which stable traveling pulses exist, we employ $\eta=+0.29$ for definiteness. This implies that $E_{2}^{ \pm}$of $q \approx \pm 8.8 \times 10^{-4}$. Since $\Gamma_{i}$ converges to 0 as $p \rightarrow \pm \infty$, the orbit converges to either $E_{2}^{+}$or $E_{2}^{-}$as $t \rightarrow \infty$ in a far field depending on the sign of $q(0)$. In fact, the far-field limit of Eq. (8) is given by its second equation without the $\Gamma_{1}$ term equivalent to the homogeneous case. The issue is to study the fate of the orbit starting from $E_{2}^{+}$at $p=-\infty$ for a given bump heterogeneity. When it goes to $E_{2}^{+}\left(E_{2}^{-}\right)$as $t \rightarrow+\infty$, it means that the pulse penetrates (rebounds from) the bump. Depending on the width and height of the bump, the orbit could be trapped by one of the pinning states around the bump, as will be discussed in the next subsection. In general, what we have to do is to find a heteroclinic connection (in a generalized sense) starting from $E_{2}^{+}$at $p=-\infty$.

Remark 2. When the heterogeneity is of Dirac- $\delta$ type, i.e., $\xi(x)=\delta(x)$, then $\Gamma_{1}(p)=\phi_{u}^{*}(-p)$ for our case, where $\phi_{u}^{*}(-p)$ denotes the u-component of $\phi^{*}$. In view of Fig. 4 , the profile of $\Gamma_{1}$ in Fig. 5 for the narrow case is consistent with this fact. Note that the u-component of the adjoint function for the Gray-Scott model decays monotonically as $|x|$ goes to $\infty$ and does not cross the $x$ axis unlike the three-component system (see Figs. 15 and 16).

\section{Comparison between PDE and ODE}

There are eight qualitatively different behaviors of Eq. (8) depending on the width $d$ and the height $\epsilon$, as shown in Fig. 6(a), which reflects the original PDE dynamics of Fig. 1(c) quite well. The aim of this section is to clarify the underlying mechanism responsible for the transitions in ODE dynamics, and then find their PDE counterparts. There appear many HIOPs as depicted in Figs. 7 and 8, which play a role of separator at each transition point. It looks obvious that there occurs penetration for sufficiently small $\epsilon$, however as $|\epsilon|$ becomes slightly larger, it is not so clear why oscillation behavior appears for positive $\epsilon$ and rebounds for negative $\epsilon$. Since the information about the width and height of the bump is encoded in the heterogeneous terms $\Gamma_{i}(i$ 

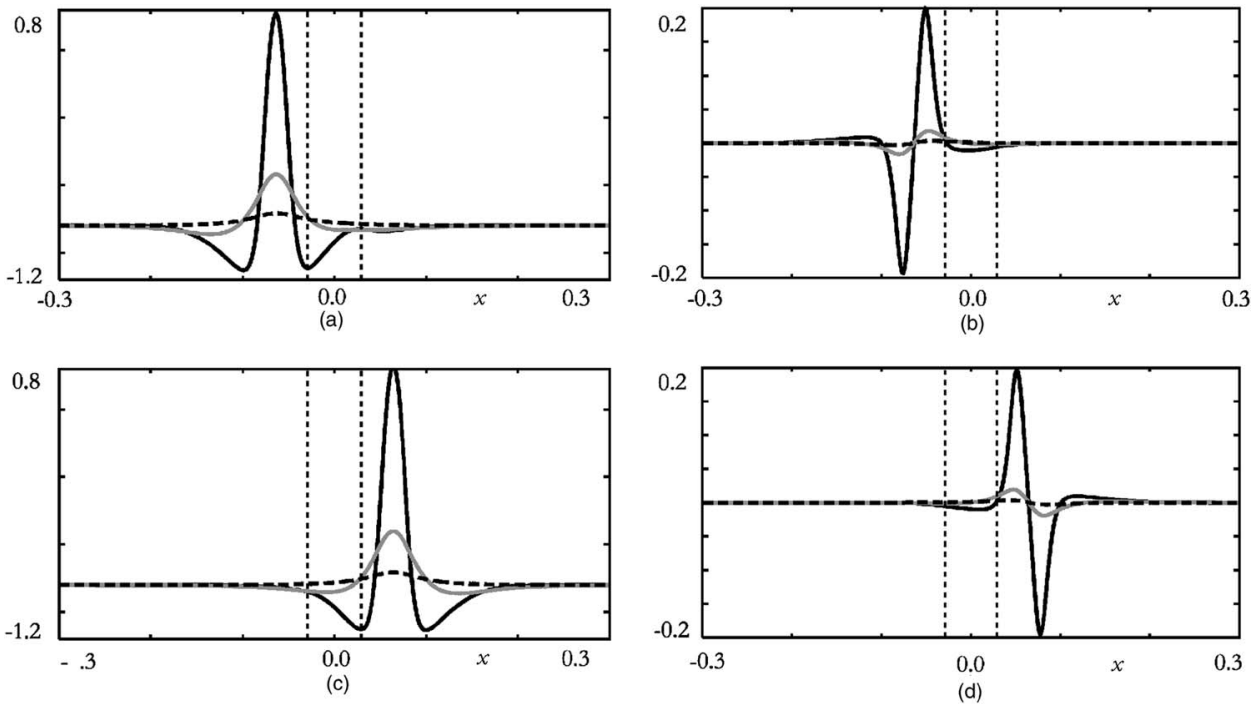

FIG. 7. The profiles of separators and the associated eigenfunctions for $(\gamma, d)=(100,0.058)$ are depicted in (a) and (b) with $\lambda \approx 0.0426$ near point A in Fig. 1(c), (c) and (d) near point $\mathrm{B}$ with $\lambda \approx 0.0262$, and (e) and (f) near point $\mathrm{C}$ with $\lambda \approx 0.0335$. The solid, gray, and broken lines represent the $u, v$, and $w$ components, respectively.
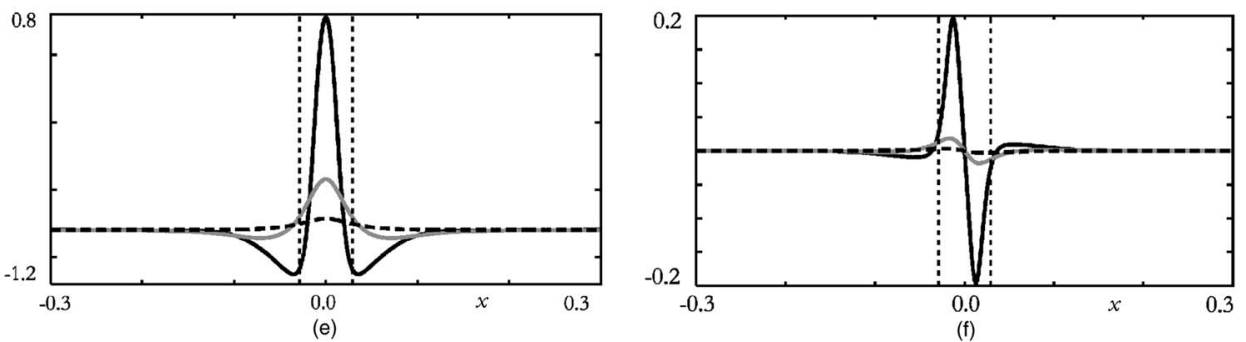

$=0,1$, we should look at their detailed profiles. Note that both $\Gamma_{i} \mathrm{~s}$ have an even number of extrema in an odd symmetric manner (see Fig. 5). For positive $\epsilon$, when the pulse comes from the left, the pulse feels like small deceleration $\rightarrow$ large acceleration $\rightarrow$ large deceleration $\rightarrow$ small acceleration and vice versa, with the opposite effect for negative $\epsilon$. Therefore, what is expected after the penetration, as $\epsilon$ is increased, is some sort of trapping between large acceleration and large
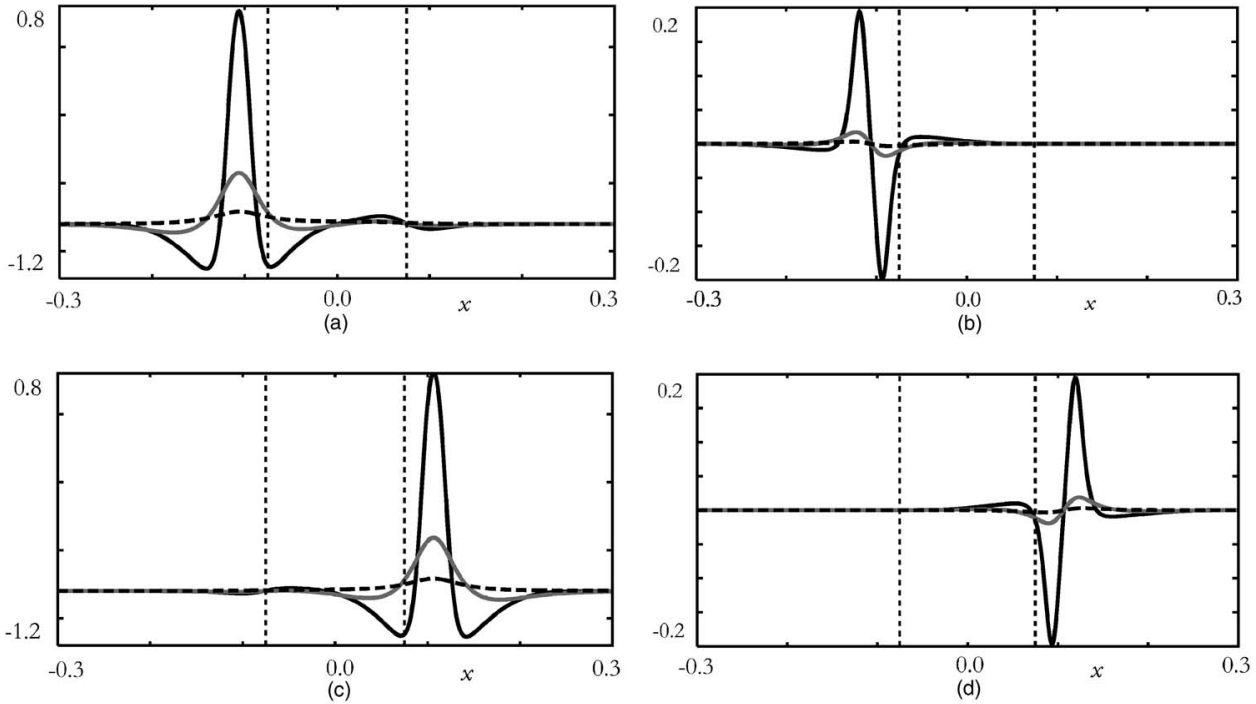

FIG. 8. The profiles of separators and the associated eigenfunctions for $(\gamma, d)=(100,0.15)$ are depicted in (a) and (b) with $\lambda \approx 0.0409$ near point $\mathrm{E}$ in Fig. 1(c), (c) and (d) near point $\mathrm{F}$ with $\lambda \approx 0.0241$, and (e) and (f) near point $\mathrm{G}$ with $\lambda \approx 0.0288$. The solid, gray, and broken lines represent $u, v$, and $w$ components, respectively.
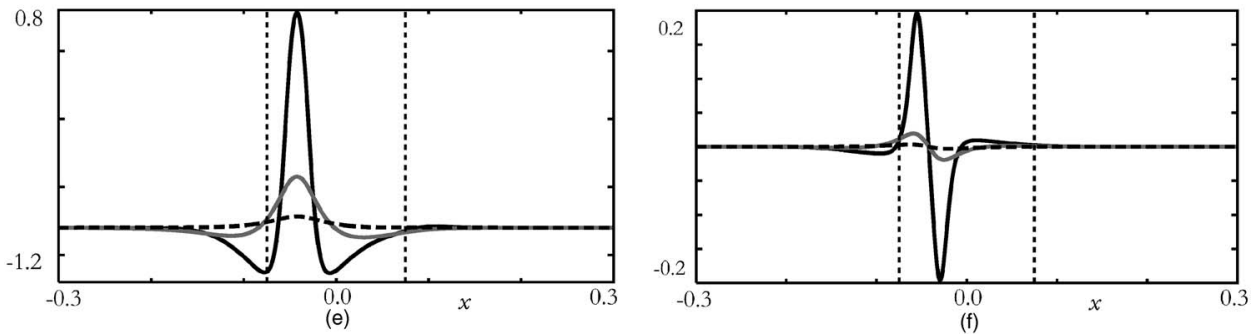


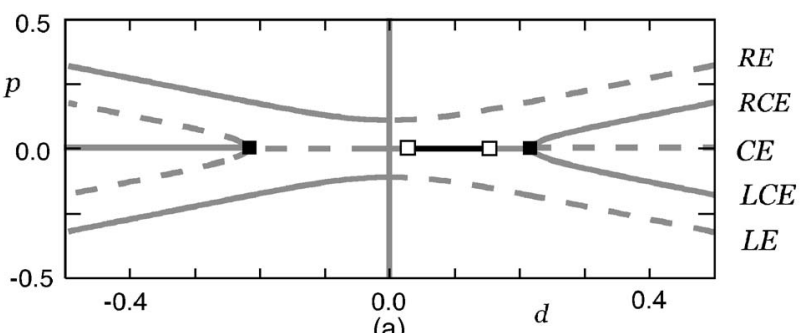

(a)

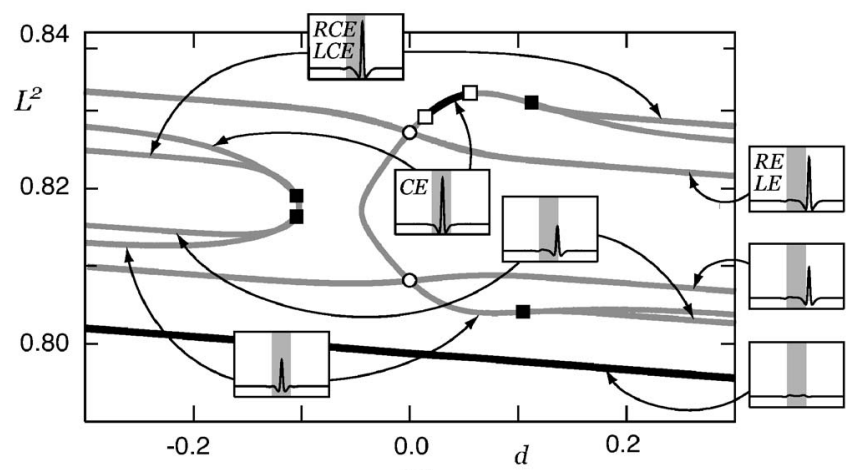

(b)

FIG. 9. (a) Bifurcation diagrams of ODE system (8) with respect to the bump width $d$ for $\epsilon=0.75$. By increasing the bump width $d$, the number of equilibrium points changes from 3 to 5 through the pitchfork bifurcation at $|d| \approx 0.21$. The black and white squares indicate the pitchfork and Hopf bifurcation points, respectively. The black and gray solid lines indicate the stable and unstable focus and the gray broken line indicates the unstable saddle, respectively. The gray line at $d=0$ indicates the translation-free zero solution. (b) Bifurcation diagram of the PDE system (1) for $\epsilon=+0.10$. Note that the branch of the center standing pulse turns back and connects to the small standing pulse via a saddle-node bifurcation. The white circles indicate the large and small standing pulses in the homogeneous case, respectively. The profiles of the $u$ component are depicted in the insets.

deceleration regions. On the other hand, for negative $\epsilon$, the second large deceleration region dominates the dynamics, and hence rebound occurs instead. More detailed analysis of ODEs allows us to explain all the dynamic transitions in a precise way. In fact, it turns out that unstable equilibria and their stable and unstable manifolds play a crucial role for our purpose. As depicted in Fig. 9 a remarkable thing is that we can find the PDE counterparts by using AUTO software as well as the Newton method. Finally, note that when we compare the two dynamics of PDE and its reduction to ODEs, their parameter values of $\gamma$ are not the same in order to have qualitatively the same dynamics, in fact $\gamma=100$ for PDE and $\gamma=30$ for ODEs, although the information on the size of the pulse is encoded in the eigenforms of Fig. 4.

\section{ODE dynamics near drift instability}

First we locate the equilibrium points of Eq. (8). Solving the first equation with respect to $q$, and substituting it into the second equation, we see that the location of the equilibrium point of Eq. (8) is determined by that of $\Gamma_{1}$ via the implicit function theorem. More precisely, any nondegenerate zero point of $\Gamma_{1}$ can be continuated to be an equilibrium point of Eq. (8) as a function of $(\epsilon, \eta)$. Recall that $\epsilon$ and $\eta$ are small quantities. The profiles of $\epsilon \approx-0.811$ are computed numerically as in Fig. 5. Note that the zero points of $\Gamma_{0}$ and $\Gamma_{1}$ almost coincide numerically except for the common zero at

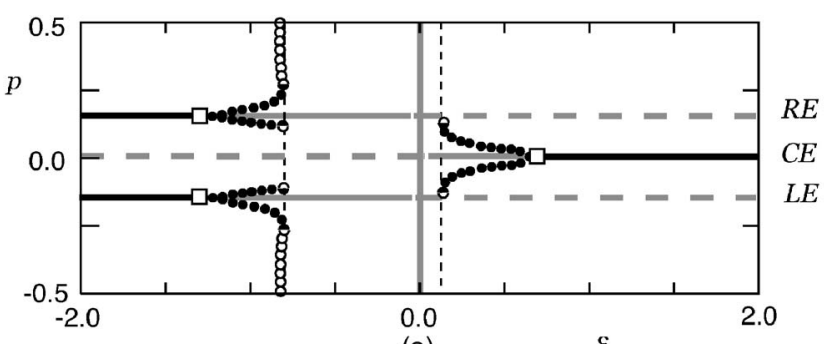

(a)

$\varepsilon$

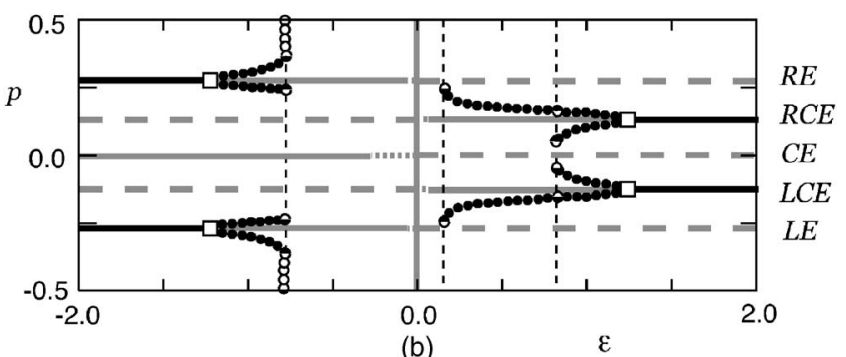

(b)

$\varepsilon$

FIG. 10. Global bifurcation diagrams of defects with respect to $\epsilon$ for ODE system (8) are depicted in (a) for the case of three zeros $d=0.15$ and (b) for the case of five zeros $d=0.40$. The positions of the equilibrium points almost do not depend on the $\epsilon$ values. The black and gray solid lines indicate the stable and unstable spirals, respectively. The gray broken and dotted lines indicate the unstable saddle and node, respectively. The white square indicates the Hopf bifurcation points. The gray lines at $\epsilon=0$ indicate the translation-free zero solutions. The black and white disks correspond to the stable and unstable limit cycles, respectively. The limiting points of the limit cycles around LE and RE are located at $\epsilon \approx-0.811$ in (a) and -0.778 in (b). The vertical broken lines indicate the location of global bifurcations for the limit cycles.

the origin due to the odd symmetry, therefore it suffices to focus on the behaviors of $\Gamma_{1}$. It is confirmed numerically that $\Gamma_{1}$ has three zeros (LE, CE, RE) for small $d$ and five zeros for large $d$ (LE, LCE, CE, RCE, RE), and a pitchfork bifurcation around $d=0.21$ at $\mathrm{CE}$ is responsible for this change. Here LCE is an abbreviation of left-center-equilibrium and similarly for other equilibria. Local stability of each equilibrium point of $(p, q)=(\bar{p}, \bar{q})$ is determined by the following linearized system:

$\left(\begin{array}{l}\dot{p} \\ \dot{q}\end{array}\right)=\left.\left(\begin{array}{cc}-\epsilon \partial_{p} \Gamma_{0} & 1 \\ \epsilon \partial_{p} \Gamma_{1} & 3 M_{1} \epsilon^{2} \Gamma_{0}^{2}+M_{2} \eta\end{array}\right)\right|_{(\bar{p}, \bar{q})}\left(\begin{array}{l}p \\ q\end{array}\right) \equiv L\left(\begin{array}{l}p \\ q\end{array}\right)$.

The eigenvalue problem $L \Psi=\lambda \Psi$ can be solved easily as $\lambda=\frac{1}{2}\left(\tau \pm \sqrt{\tau^{2}-4 \Delta}\right)$, where $\tau=3 M_{1} \epsilon^{2} \Gamma_{0}^{2}+M_{2} \eta-\epsilon \partial_{p} \Gamma_{0}$ and $\Delta=-\epsilon \partial_{p} \Gamma_{0}\left(3 M_{1} \epsilon^{2} \Gamma_{0}^{2}+M_{2} \eta\right)-\epsilon \partial_{p} \Gamma_{1}$. The associated eigenvectors $\Psi$ are obtained in the directions of $(2, \tau$ $\left.+2 \epsilon \partial_{p} \Gamma_{0} \pm \sqrt{\tau^{2}-4 \Delta}\right)$. For small $\eta$ and $\epsilon$, the dominant terms of the trace $\tau$, determinant $\Delta$, and discriminant $\tau^{2}-4 \Delta$ are given by $M_{2} \eta-\epsilon \partial_{p} \Gamma_{0},-\epsilon \partial_{p} \Gamma_{1}$, and $4 \epsilon \partial_{p} \Gamma_{1}$, respectively. In view of the profiles of Fig. 5, especially the sign of the derivatives at the zero points of $\Gamma_{i}$, the stability and bifurcation diagram with respect to the height $\epsilon$ at each equilibrium point can be computed as in Fig. 10. When $\epsilon$ changes its sign, so does $\Delta$, therefore the transition saddle (gray broken) $\rightarrow$ node (gray dotted) $\rightarrow$ spiral (gray solid) or vice versa is basically observed, although the node regime is very narrow compared with others. Also when the size of $\epsilon$ becomes larger in modulus, the trace $\tau$ changes its sign. Therefore, 
there always occurs Hopf bifurcation along each equilibrium for either positive or negative $\epsilon$ depending on the sign of $\partial_{p} \Gamma_{0}$. All the Hopf bifurcations are supercritical and hence stable limit cycles are observed at least locally. Global behaviors of those Hopf branches are also depicted in Fig. 10. The period of each branch emanating from either LE or RE goes to infinity as the cycle approaches a homoclinic orbit via the saddle-node point, the details of which will be explained later. For the five zeros case, the two Hopf branches from LCE and RCE merge via figure-eight bifurcation around $\epsilon \approx 0.822$ and become a larger Hopf cycle, which eventually deforms into a heteroclinic cycle. We will discuss the details in the sequel and clarify how these behaviors are relevant to the transitions of pulse dynamics as explained in the next subsection.

\section{For the narrow bump case of $d=0.15$ (three zeros)}

First, we study the case of the narrow bump (three zeros) for $(\gamma, d)=(30,0.15)$, with particular focus on the dynamics near four transition points A, B, C, and D in Fig. 6(a). The associated flows are depicted in Figs. 6(b)-6(e). For positive $\epsilon$, we have the following three transitions, i.e., REB $1 \rightarrow$ STA $1 \rightarrow$ OSC $1 \rightarrow$ PEN. In view of Fig. 6, the first transition from REB1 to STA1 occurs at $\epsilon \approx 0.916$ near point A, and the (left-upper) stable manifold of the LE point plays a role as separator between REB1 and STA1. Recall that the first transition A in PDE dynamics [see Fig. 1(c)] is also from REB1 to STA1 as in Figs. 2(a) and 2(b) around $\epsilon$ $\approx 0.16470$. The plot of the peak location and its velocity of Fig. 2(a) is qualitatively the same as Fig. 6(b). It is clearly seen in Fig. 2(a) that the solution takes a quasisteady state for a certain time before reaching the bump region, after which it either rebounds or settles down to the standing pulse located at the center of the bump. It is numerically confirmed that the quasisteady state turns out to be a standing pulse of codim1 located slightly to the left of the jump point as depicted in Figs. 7(a) and 7(b), which corresponds to the saddle point LE in ODE dynamics. The standing pulse located at the center of the bump is the PDE counterpart of the equilibrium $\mathrm{CE}$ and it plays a role of separator with its stable manifold in PDE dynamics. The PDE separator has only one positive eigenvalue of $\lambda \approx 0.0426$, and the associated eigenform is shown in Fig. 7(b). Before going to transition B, we remark on the transition from STA1 to OSC1 in Fig. 6(a). This is simply due to the Hopf bifurcation of $\mathrm{CE}$ at $\epsilon \approx 0.66$ as in Fig. 10(a). The attractor is switched to the limit cycle around CE. The exact same thing happens in PDE dynamics as in Fig. 9(b). Therefore, ODE dynamics captures the essence of the PDE dynamics for this transition. The emanating limit cycle becomes larger and approaches the heteroclinic cycle connecting $\mathrm{LE}$ and $\mathrm{RE}$ as $\epsilon$ becomes closer to the transition point $\mathrm{B}$ at $\epsilon \approx 0.143$ as depicted in Fig. 10(a). As $\epsilon$ is decreased, the heteroclinic cycle disappears at $\epsilon \approx 0.141$ and the orbit penetrates the bump region as shown in Fig. 6(c). This shows that depinning occurs due to the onset of heteroclinic bifurcation connecting LE to RE; in fact, a double heteroclinic loop appears because of the symmetry of the ODE system. This is noticed also in Ref. 43 for the twocomponent system of FitzHugh-Nagumo type. The separator between OSC1 and PEN is therefore the (upper) stable manifold of the equilibrium RE. This explains the PDE transition B as clearly shown in Figs. 2(c) and 2(d); in fact, the quasisteady state observed is confirmed as Figs. 7(c) and 7(d), which are the PDE counterpart of RE. Note that the location of this standing pulse is slightly outside of the right edge of the bump. Hence ODE dynamics inherits the information from PDE dynamics not only about the existence of the separator but also its location.

For the negative $\epsilon$ region, we have the following three transitions: PEN $\rightarrow$ REB2 $\rightarrow$ OSC2 $\rightarrow$ STA2. The separator between PEN and REB2 is the (upper) stable manifold of the CE point at $\epsilon \approx-0.136$ near C in Fig. 6(a). The codim 1 separator whose peak position is the center of the bump domain can be confirmed by numerics in the original PDE system, as shown in Figs. 7(e) and 7(f).

Transition form OSC2 to REB2 near D occurs by the homoclinic bifurcation (each limit cycle around LE and RE becomes a homoclinic orbit to the saddle point $E_{1}$ located at $p= \pm \infty$, respectively) at $\epsilon \approx-0.823$. In addition, a saddlenode bifurcation occurs for the limit cycle branch at $\epsilon$ $\approx-0.811$ as in Fig. 10(a). The unstable oscillatory basins becomes increasingly angled and the cycle passes closer and closer to the saddle point of $E_{1}$ as $\epsilon$ approaches the transition point $\mathrm{D}$ from below. This homoclinic orbit is stable from the inside, because of the supercritical Hopf bifurcation at LE around $\epsilon \approx-1.25$, which is responsible for the transition from STA2 to OSC2.

\section{For the wide bump case of $d=0.40$}

There are seven different dynamic regimes from REB1 to STA2 for the wide bump (five zeros) case of $(\gamma, d)$ $=(30,0.40)$ as $\epsilon$ is decreased. The associated flows are depicted in Fig. 11. Due to the pitchfork bifurcation at CE as $d$ is increased, $\mathrm{CE}$ becomes a saddle for all positive $\epsilon$ and changes to an unstable node and spiral for negative $\epsilon$ as shown in Fig. 10(b). On the other hand, the resulting two equilibria LCE and RCE branching from $\mathrm{CE}$ are spirals for positive $\epsilon$ and become saddles for negative $\epsilon$. Two supercritical Hopf bifurcations occur simultaneously at these two equilibria around $\epsilon \approx+1.24$, and those cycles coexist up to the global bifurcation as in Fig. 10(b), the details of which are described below. It should be noted that the same type of bifurcation occurs for the PDE system as shown in Fig. 9(b): two standing pulses bifurcate from the central standing pulse around $d \approx 0.10$, which are the PDE counterparts of LCE and RCE.

For positive $\epsilon$, we have the following three transitions as $\epsilon$ is decreased: REB $1 \rightarrow$ OSC $3 \rightarrow$ OSC $1 \rightarrow$ PEN. Note that there appear two different oscillatory regions: one is a set of two small cycles rotating around LCE and RCE, respectively, and the other is a large cycle enclosing three equilibria LCE, CE, and RCE as shown in Figs. 11(a)-11(c). The transition from REB1 to OSC3 near E $(\epsilon \approx 0.858)$ is controlled by the (upper) stable manifold of LE as clearly seen from Fig. 11(a), namely LE is the separator for this transition. As $\epsilon$ is decreased, these two cycles become larger and deform into a large limit cycle of peanut shape enclosing the three equilibrium points of $\mathrm{LCE}, \mathrm{CE}$, and $\mathrm{RCE}$ via a figure-eight bifur- 

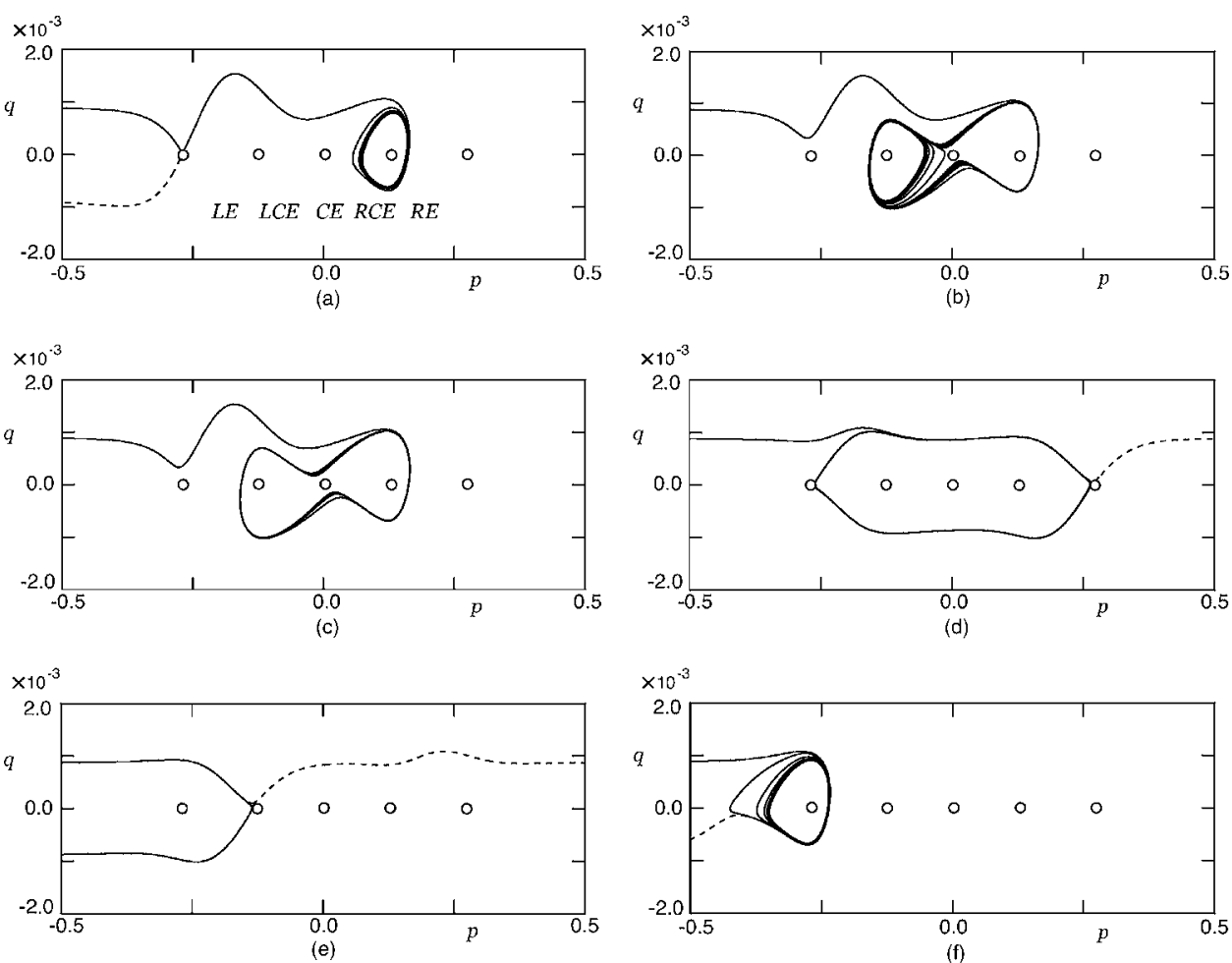

FIG. 11. Orbit flows in $(p, q)$ space for the wide bump case of $(\gamma, d)$ $=(30,0.40)$. (a) Phase boundary between REB1 (dotted line) and OSC3 (solid line) at $\epsilon \approx 0.858$ near point $\mathrm{E}$ in Fig. 6(a). Transition from (b) OSC3 to (c) OSC1 occurs at $\epsilon$ $\approx 0.825$ via a saddle-node bifurcation for the large limit cycle branch. There remains a temporary oscillatory motion as an aftereffect of the limiting point. (d) Transition from OSC1 (solid line) to PEN (dotted line) occurs at $\epsilon \approx 0.157$ near point $\mathrm{F}$. (e) Phase boundary between PEN (dotted line) and REB2 (solid line) at $\epsilon \approx-0.158$ near point $G$. (f) Transition from REB2 (dotted line) to OSC2 (solid line) occurs at $\epsilon \approx-0.787$ near point $H$. The open circles indicate the unstable stationary states. cation as depicted in Figs. 12(a) and 12(b). The detailed manner of transition from OSC3 to OSC1 is rather complicated; in fact, two small cycle branches are not reconnected directly to the large cycle branch through figure-eight bifurcation, but it is bent twice. In other words, there are two saddle-node points, each of which belongs to OSC3 or OSC1 type, and figure-eight bifurcation occurs at $\epsilon \approx 0.822$ on the middle unstable branch as shown in Fig. 12(b). Note that these two saddle-node points are so close each other that they are not visible in Fig. 10(b). The transition from OSC3 to OSC1 is due to the existence of the limiting point at $\epsilon$ $\approx 0.825$ for the large cycle branch. The orbital behavior after that displays an aftereffect of the saddle-node point, in which there remains a temporary large oscillatory motion as shown in Fig. 11(b). Note that the other limiting points for limit cycles around LCE and RCE are located at $\epsilon \approx 0.816$. In view of the PDE phase diagram of Fig. 1(c), the dynamics OSC3 is not observed, and only the transition from REB to OSC1 is detected as depicted in Figs. 3(a) and 3(b). Namely, we could not find a small-width oscillatory pulse moving around only in the left or right portion of the bump region. There might be a possibility to find such an oscillatory pulse when the width of the bump and the system size become larger simultaneously, however at present it remains an open question. On the other hand, the PDE counterpart of LE for the transition from REB to OSC1 is numerically detected as in Figs. 8(a) and 8(b), the location of which is slightly outside of the left edge of the bump. The large limit cycle becomes larger as $\epsilon$ is decreased, and approaches a double heteroclinic loop connecting LE and RE at $\epsilon \approx 0.157$, which is responsible for the transition from OSC1 to PEN near point F. The large limit cycle disappears below this global bifurcation point, and only penetration is observed. This ex- plains quite well the transition in PDE setting as shown in Figs. 3(c) and 3(d). For negative $\epsilon$, we have the following four regimes: PEN, REB2, OSC2, and STA2. The transitions among them can be explained in the same way as the narrow width case, therefore we only sketch it briefly. The (upper) stable manifold of the LCE point plays a role as a separator between PEN and REB2 at $\epsilon \approx-0.158$ near point $G$. The PDE counterpart is shown in Figs. 3(e) and 3(f) (dynamics) and the separator in Figs. 8(e) and 8(f). The (upper) stable manifold of the standing pulse $E_{1}$ at $p=-\infty$ plays a key role as a separator between REB2 and OSC2 at $\epsilon \approx-0.787$ near point D.

Transition from REB2 to OSC2 occurs by the homoclinic bifurcation at $\epsilon \approx-0.787$ near point $H$. In addition, a saddle-node bifurcation occurs for the limit cycle branch at $\epsilon \approx-0.778$. Qualitatively similar dynamics is also observed for PDE dynamics as depicted in Figs. 3(g) and 3(h). The schematic orbital behaviors near the transition from OSC2 to REB2 are depicted in Fig. 12(c). The supercritical Hopf bifurcation of the LE point causes the transition from OSC2 to STA2. A similar Hopf bifurcation also occurs for the standing pulse located at the center of the bump (not shown here). Overall the reduced ODE dynamics almost completely describes the PDE behavior when the pulse meets the heterogeneity.

Remark 3. We consider so far only the symmetric bump, namely $\chi(x)$ has a reflectional symmetry at origin. For the asymmetric case, the analysis in this section can be done in a parallel way, in fact the imperfections occur to the bifurcation diagram of Fig. 10 and, hence, right- and left-going pulses have different behaviors in general. More detailed study will be reported elsewhere. 


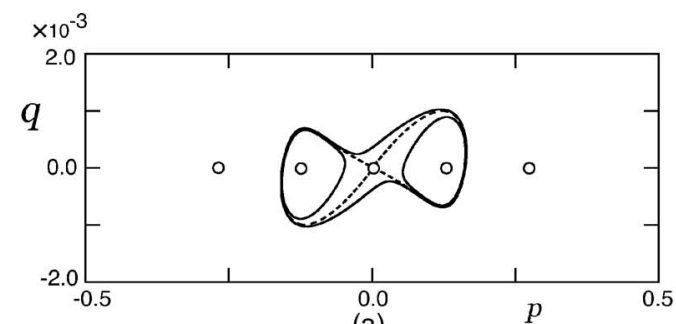

(a)

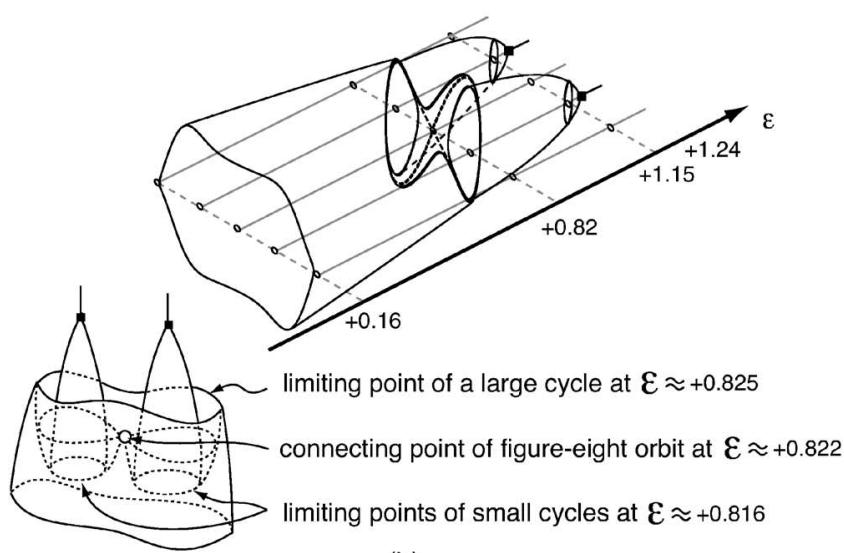

(b)
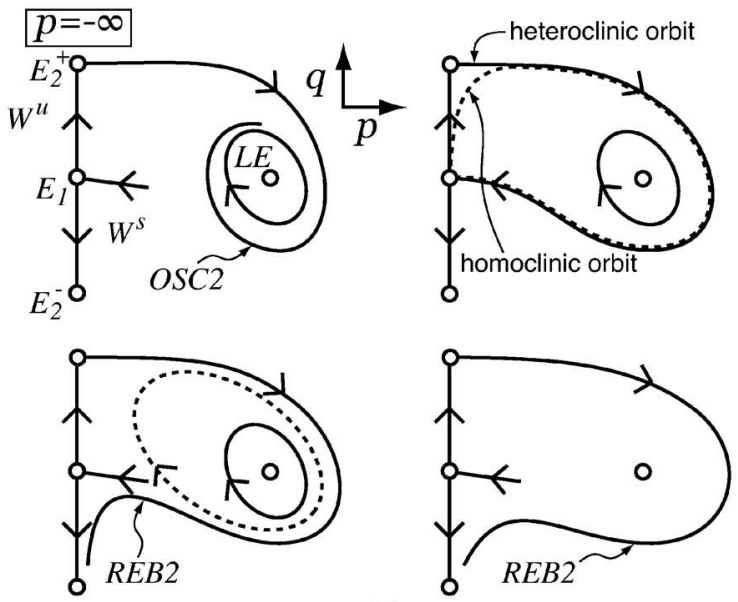

(c)

FIG. 12. (a) Coexistence between the oscillatory solutions at the figure-eight bifurcation point at $\epsilon \approx 0.822$ near point $\mathrm{E}$. The solid lines and dotted line show the stable limit cycles and the unstable figure-eight orbit, respectively. (b) The whole structure of the limit cycles with respect to $\epsilon$ forms a surface similar to trousers. Each bottom of the oscillatory solutions emanates from the supercritical Hopf bifurcation points at $\epsilon \approx 1.24$, and they are connected around $\epsilon \approx 0.822$. The connected limit cycle disappears by the heteroclinic bifurcations at $\epsilon \approx 0.157$. The large cycle and small cycles lose the stability via saddle-node bifurcations at $\epsilon \approx 0.825$ and 0.816 , respectively. A figureeight solution connects those unstable limit cycles by figure-eight bifurcation. (c) The schematic orbit flows near the phase boundary between OSC2 and REB2 from left-top to right-bottom pictures. A homoclinic orbit to the saddle $E_{1}$ belongs to the intersection of unstable and stable manifolds of $E_{1}$. An unstable limit cycle (dotted line) appears from the homoclinic bifurcation and it disappears with the stable one by saddle-node bifurcation, as $\epsilon$ is increased. The white circles indicate the unstable equilibrium points.

\section{THE GRAY-SCOTT MODEL FOR BUMP AND PERIODIC MEDIA (DEFECTLESS)}

In the preceding sections, we dealt with pulse dynamics near codim1 point with drift instability. In this section, we investigate pulse dynamics with codim 2 singularity consist-

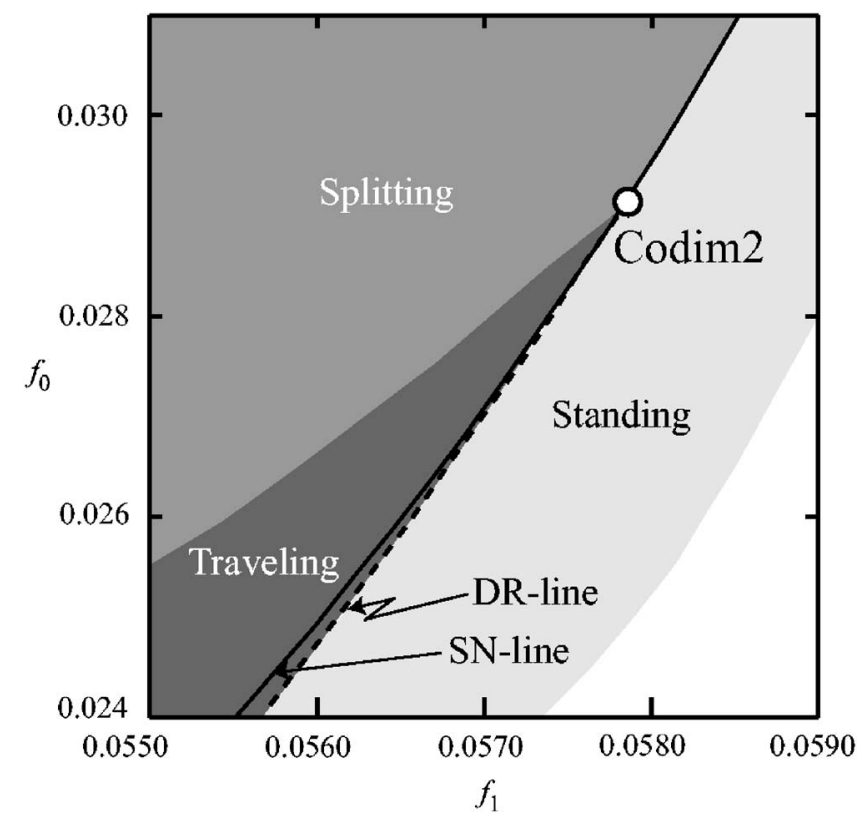

FIG. 13. The phase diagram in $\left(f_{0}, f_{1}\right)$ space for the homogeneous system. The black and light gray regions indicate stable traveling and standing pulses, respectively. In the dark gray region, the splitting behavior is observed. The triple junction of these three regions is located at $(0.0291,0.0578)$, which coincides with the codim2 point. The solid (dotted) line stands for the saddle-node (drift) bifurcation of the standing pulse.

ing of drift and saddle-node bifurcations. For definiteness, we employ the following Gray-Scott model:

$$
\begin{aligned}
& u_{t}=D_{u} \Delta u-u v^{2}+f_{0}(1-u), \\
& \tau v_{t}=D_{v} \Delta v+u v^{2}-\left(f_{0}+f_{1}\right) v,
\end{aligned}
$$

where $u=u(t, x), v=v(t, x)$ depend on time $t$ and $x \in \mathbb{R}^{1}$. It was studied in Ref. 14 that the standing pulse solution of Eq. (9) has both drift bifurcation (DR) and saddle-node bifurcation (SN) and they can occur simultaneously in twoparameter space $\left(f_{0}, f_{1}\right)$. Actually, such a codim 2 point is numerically detected at $\left(f_{0}, f_{1}\right) \approx(0.0291,0.0578)$, and all kinds of patterns are observed around it, as shown in Fig. 13. Stable traveling solutions emanate via supercritical drift bifurcation, and pulse splitting is observed via saddle-node bifurcation. Now we introduce a heterogeneity to the parameter $f_{1}$ as $f_{1}(x)=f_{1}+\epsilon \chi(x)$.

As a heterogeneity, we employ the bump-type function (2). In this section, we take $f_{0}$ and $f_{1}$ near the codim 2 point and $D_{u}=4.0 \times 10^{-5}, D_{v}=2.0 \times 10^{-5}$, and $\tau=1.007734$. The heterogeneity $\chi(x)$ takes the same functional form as Eq. (2).

Pulse dynamics of Eq. (9) for a single jump case near the codim 2 point was studied in Ref. 29. One of the differences between jump and bump cases is the existence of HIOP, i.e., there exist unstable stationary standing pulse solutions located at the center for the bump case while no stationary standing pulse solution exists for the jump case in Ref. 29.

We show that Eq. (9) has qualitatively three different outputs for positive $\epsilon$ : penetration, rebound, and splitting. We derive then ODEs near the codim 2 point and investigate the underlying structure responsible for the transitions among those outputs. It turns out that a heteroclinic orbit connecting 


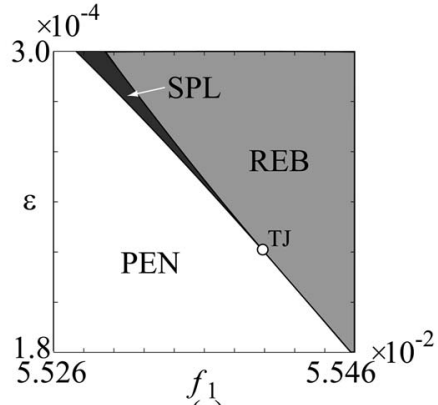

(a)

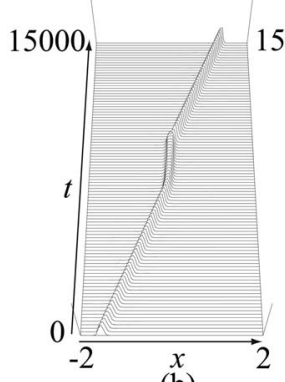

(b)

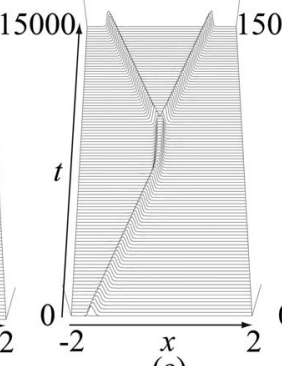

(c)

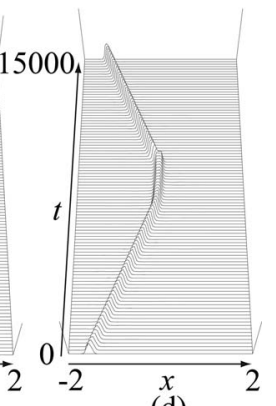

(d)
FIG. 14. (a) The phase diagram of Eq. (9) for $\gamma=100$ and $d=0.2$. PEN: penetration; REB: rebound; SPL: splitting. TJ is a triple junction of these regions. (b)-(d) Pulse dynamics near TJ for $\epsilon=2.14 \times 10^{-4}$. (b) Penetration $\left(f_{1}=5.54084700 \times 10^{-2}\right)$. (c) Splitting $\left(f_{1}=5.54084705 \times 10^{-2}\right)$. (d) Rebound $\left(f_{1}=5.54084710 \times 10^{-2}\right)$.

two HIOPs plays a pivotal role to control the traffic of orbits. Finally, we present a complex behavior of pulses for periodic heterogeneities as well as long- and short-wave limits.

\section{A. Phase diagram of PDE dynamics}

The heterogeneous function (2) is fixed to be $\gamma=100$ and $d=0.2$, since it is numerically confirmed that the pulse dynamics does not depend on the width $d$ of the bump for the Gray-Scott model. We employ $f_{1}$ and $\epsilon$ as control parameters with $f_{0}=0.024$. We only consider the case for $\epsilon>0$. Note that the pulse is heading to the bad environment for $\epsilon>0$ in the sense that traveling velocity becomes smaller inside of the bump. We find by numerics that there are three different outputs for Eq. (9): penetration (PEN), rebound (REB), and splitting (SPL) [see Fig. 14(a)]. The SPL region has a tongue shape and the tip of it touches at the triple junction (TJ) where $\left(f_{1}, \epsilon\right)=\left(f_{\mathrm{TJ}}, \epsilon_{\mathrm{TJ}}\right) \approx\left(0.0554,2.13 \times 10^{-4}\right)$ [Fig. 14(a) . Hence when $f_{1}>f_{\mathrm{TJ}}$, the dynamics changes from penetration to rebound as increasing $\epsilon$ from 0 . When $f_{1}>f_{\mathrm{TJ}}$, the dynamics changes penetration $\rightarrow$ splitting $\rightarrow$ rebound as $\epsilon$ is increased. The orbital behaviors near TJ are depicted in Figs. 14(b)-14(d). A common feature is that each orbit takes a quasisteady state for a certain time, then starts to deform depending on the parameter value. A detailed structure will be revealed through an analysis of ODEs and global bifurcational behaviors of HIOPs.

\section{B. Reduction to ODEs with codim2 singularity}

In order to clarify the orbital behaviors near TJ, we study the following finite-dimensional system near the codim2 point with heterogeneity. A sketch of its derivation is given
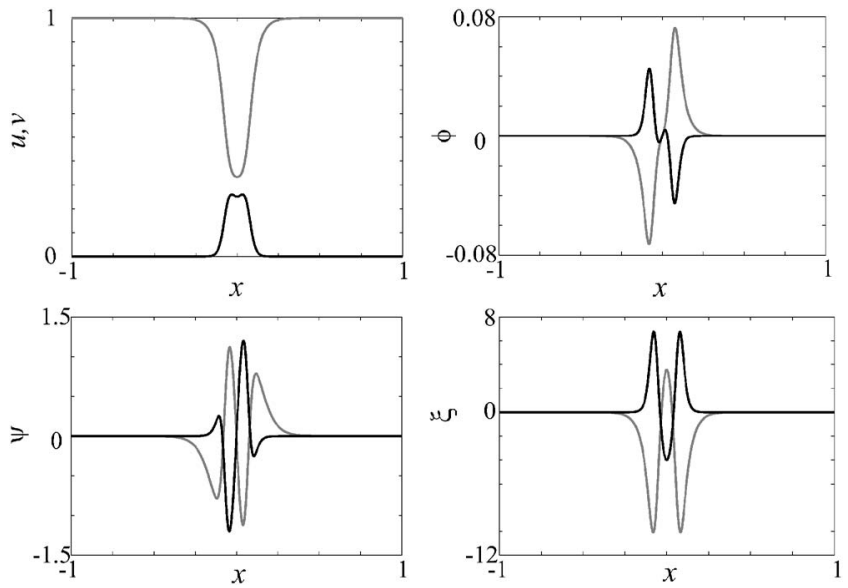

FIG. 15. Profiles of stationary solutions and the associated eigenfunctions at the codim 2 point. $\phi, \psi$, and $\xi$ correspond to translation-free, drift bifurcation, and saddle-node bifurcation, respectively. The solid (gray) line indicates the $v$ component ( $u$ component).

in the Appendix, although the details are delegated to Ref. 29. The principal part of Eq. (A6) is given by

$$
\begin{aligned}
& \dot{p}=\rho / C_{2}, \quad \dot{\rho}=\rho\left(s \mu_{1}+\theta_{1} \omega\right)+\epsilon \Gamma_{1}(p), \\
& \dot{\omega}=\mu_{2}+\omega^{2}-\theta_{2} \rho^{2}+\epsilon \Gamma_{2}(p),
\end{aligned}
$$

where $\mu_{1}$ and $\mu_{2}$ are the rescaled parameters of $\left(\eta_{1}, \eta_{2}\right)$ that measure the distance from the codim 2 point TJ. We fix $\left|\mu_{1}\right|$ to be sufficiently small and take $\mu_{2}=O\left(\left|\mu_{1}\right|^{2-\nu}\right)$ and $\epsilon$ $=O\left(\left|\mu_{1}\right|^{2-\nu}\right)(2 / 3<\nu<1)$. In what follows, we employ $\mu_{2}$ as a bifurcation parameter with $\mu_{1}$ being fixed appropriately. We can still capture the essential part of the dynamics under this setting. All other coefficients are determined by the profile of the standing pulse solution of Eq. (9) at codim2 point (see the Appendix for details). Figure 15 shows the profile and the associated eigenfunctions. Note that we can drop the $\rho \omega$ term and $\epsilon \Gamma_{0}$ from the first equation, since the number of equilibrium points and their stability do not change qualitatively. Here parameters are set to $C_{2}=0.85, s=-1, \theta_{1}$ $=0.93, \theta_{2}=0.11$, and $\mu_{1}=-0.1$. We note that $t$ and $\epsilon$ in Eq. (10) are also rescaled, so they are different from those in Eq. (9). The right-hand side of the first equation $\rho$ stands for the velocity of the pulse and $\omega$ for the depth of the dimple of it related to splitting. The heterogeneous terms $\Gamma_{1}$ and $\Gamma_{2}$ are shown in Fig. 16. $\Gamma_{1}\left(\Gamma_{2}\right)$ is an odd (even) function, respectively, which comes from the fact that eigenfunctions associ-

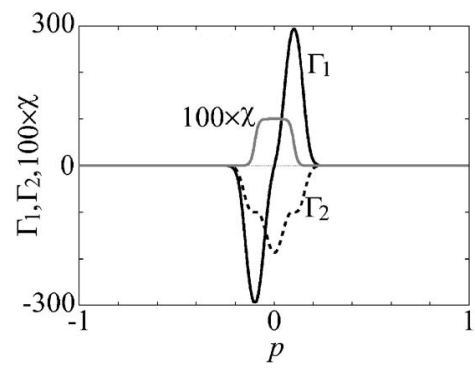

FIG. 16. Profiles of $\Gamma_{1}$ and $\Gamma_{2}$. $\Gamma_{1}$ is an odd function and has a vanishing point at $p=0 . \Gamma_{2}$ is an even function and is negative for any $p$. 


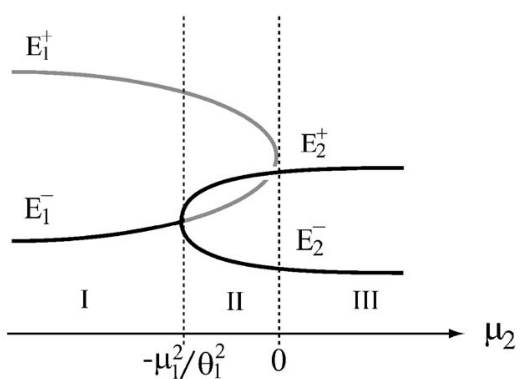

(a)

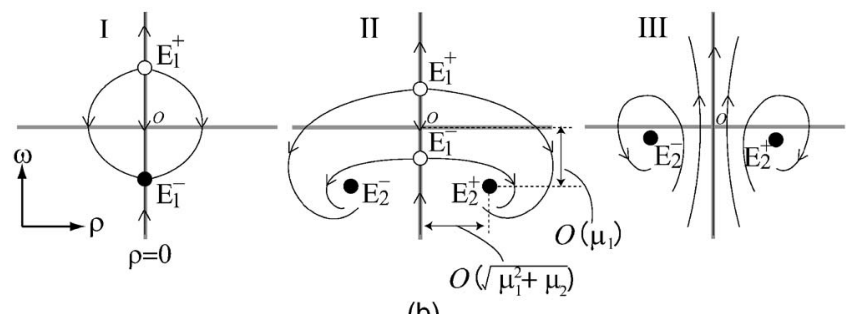

(b)

FIG. 17. (a) Schematic bifurcation diagram of Eq. (11). The solid and gray lines indicate stable and unstable branches, respectively. (b) The phase portrait of Eq. (11) with fixed $s \mu_{1}>0$. The solid and white circles indicate stable and unstable equilibria, respectively. In region II, the unstable manifolds emanating from $E_{1}^{ \pm}$are connected to $E_{2}^{ \pm}$.

ated with drift and saddle-node bifurcations have the same symmetry. We assume the following for $\Gamma_{j}$, which is numerically confirmed as in Fig. 16:

(H1) $\Gamma_{1}(0)=0$ and $\Gamma_{1}(p) \neq 0$ for $p \neq 0 . \Gamma_{2}(p)<0$ for any $p$.

(H2) $\Gamma_{j}(p)$ decays exponentially to 0 as $p \rightarrow \pm \infty$.

First we study the homogeneous case, i.e., $\epsilon=0$. Since $p$ is independent of the second and third equations, Eq. (10) is reduced to the following two-component system:

$$
\dot{\rho}=\rho\left(s \mu_{1}+\theta_{1} \omega\right), \quad \dot{\omega}=\mu_{2}+\omega^{2}-\theta_{2} \rho^{2} .
$$

Figure 17 shows a schematic bifurcation diagram of stationary solutions of Eq. (11) with respect to $\mu_{2}$.

There exist two equilibrium points for Eq. (11) with $\rho=0$,

$$
E_{1}^{ \pm}:(\rho, \omega)=\left(0, \pm \sqrt{-\mu_{2}}\right)
$$

for $\mu_{2} \leq 0 . E_{1}^{ \pm}$correspond to the standing pulse solutions of Eq. (9). These equilibria disappear by saddle-node bifurcation at $\mu_{2}=0 . E_{1}^{-}$loses its stability at $\mu_{2}=-\mu_{1}^{2} / \theta_{1}^{2}$ via a drift bifurcation. In our parameter setting, drift bifurcation occurs supercritically, i.e., two stable equilibrium solutions $E_{2}^{ \pm}$ emerge at the drift point where

$$
E_{2}^{ \pm}:(\rho, \omega)=\left( \pm \sqrt{\left(\mu_{2}+\mu_{1}^{2} / \theta_{1}^{2}\right) / \theta_{2}},-s \mu_{1} / \theta_{1}\right) .
$$

We next consider the heterogeneous case, i.e., $\epsilon>0$. When $-\mu_{2}-\epsilon \Gamma_{2}(0)>0$, Eq. (10) has only two equilibrium points (see Fig. 19),

$$
\widetilde{E}_{1}^{ \pm}:(p, \rho, \omega)=\left(0,0, \pm \sqrt{-\mu_{2}-\epsilon \Gamma_{2}(0)}\right) .
$$

It follows from $(\mathrm{H} 1)$ that there exist no equilibrium points with $\rho=0$ except for $\widetilde{E}_{1}^{ \pm}$, which make a contrast to the three-component system (1); the number of zero points of $\Gamma_{1}$

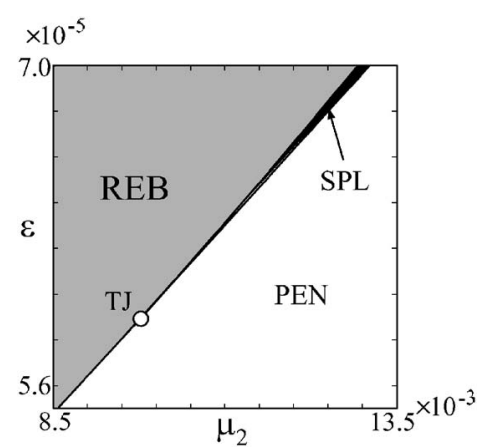

FIG. 18. Phase diagram for Eq. (10). There are three qualitatively different regimes: Penetration (PEN), Rebound (REB), and Splitting (SPL). TJ is a triple junction where PEN, REB, and SPL regions merge at $\left(\mu_{2}, \epsilon\right) \approx(9.78$ $\left.\times 10^{-3}, 5.9 \times 10^{-5}\right)$.

for it does change depending on the width of the bump. Since $\Gamma_{j}(p) \rightarrow 0$ as $p \rightarrow \pm \infty$, there exist $E_{2}^{ \pm}$located at $p$ $= \pm \infty$, which have a large basin of attraction. In fact, the orbit starting from $(\rho, \omega)$ with $\rho(0)>0[\rho(0)<0]$ approaches $E_{2}^{+}$ $\left(E_{2}^{-}\right)$as $t \rightarrow+\infty$ for sufficiently large (small) $p(0)$. Note that $\widetilde{E}_{1}^{ \pm}$converge to $E_{1}^{ \pm}$as $\epsilon \rightarrow 0$, and unstable manifolds emanating from $\widetilde{E}_{1}^{+}$connect to $\widetilde{E}_{1}^{-}$and $\omega=+\infty$, respectively.

\section{Dynamics of ODEs near TJ}

Based on the above discussions, we give the definitions of penetration, splitting, and rebound in terms of ODEs.

Definition 1. When $(\rho, \omega)$ converges to $E_{2}^{+}$as $t \rightarrow+\infty$, the dynamics is called penetration.

Definition 2. When $\omega>\delta$ for some finite $\delta$, the dynamics is called splitting.

Since we study Eq. (10) in the neighborhood of the codim 2 point, the size of $(\rho, \omega)$ is small and hence the constant $\delta$ in the above definition can be taken to be an arbitrary finite number. Hereafter we take $\delta=1$.

Definition 3. When $(\rho, \omega)$ converges to $E_{2}^{-}$as $t \rightarrow+\infty$, the dynamics is called rebound.

Figure 18 shows the phase diagram of Eq. (10) with respect to $\mu_{2}$ and $\epsilon$. It essentially inherits the original PDE dynamics of Fig. 14, namely there exist penetration, splitting, and rebound regions, and a splitting region exists in between the penetration and the rebound regions. Moreover, there exists a TJ at $\left(\mu_{2}, \epsilon\right)=\left(\mu_{\mathrm{TJ}}, \epsilon_{\mathrm{TJ}}\right) \approx\left(9.78 \times 10^{-3}\right.$, $\left.5.9 \times 10^{-5}\right)$. We show that there appear two different types of HIOPs at TJ, and they play a key role for the traffic control of orbits near TJ.

What we have to do is to classify the behaviors of pulse dynamics in heterogeneous media starting far left on the $x$ axis. Recalling that a stable traveling pulse (STP) corresponds to the equilibrium point $E_{2}^{+}$, it is natural to employ the initial data as $(\rho(0), \omega(0))=E_{2}^{+}$and $p(0)=-1.0$. As parameters are varied appropriately close to TJ, we may detect the orbit that crosses the 1D stable manifold of $\widetilde{E}_{1}^{+}$in 3D space by two-parameter searching. In the sequel, we investigate the orbital behaviors by varying $\left(\mu_{2}, \epsilon\right)$ near TJ from the left $\left(\mu_{2}<\mu_{\mathrm{TJ}}\right)$ and the right $\left(\mu_{2}>\mu_{\mathrm{TJ}}\right)$ side of it, and show the 


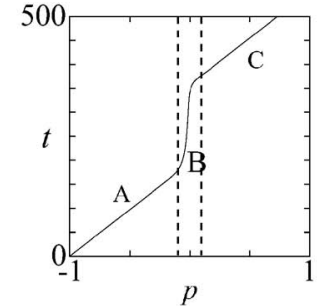

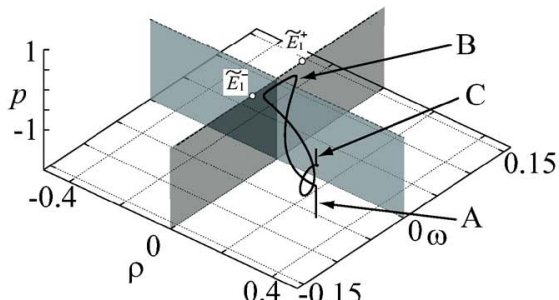

(a)

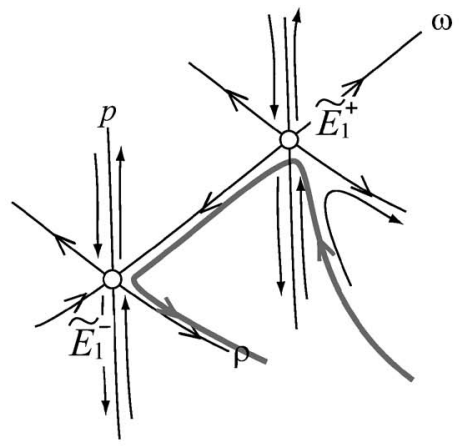

PEN
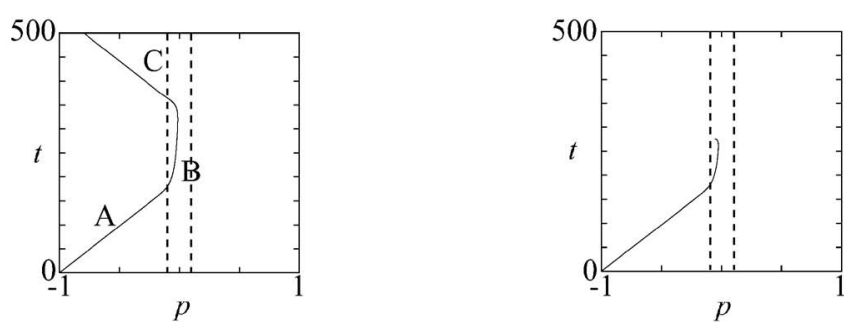

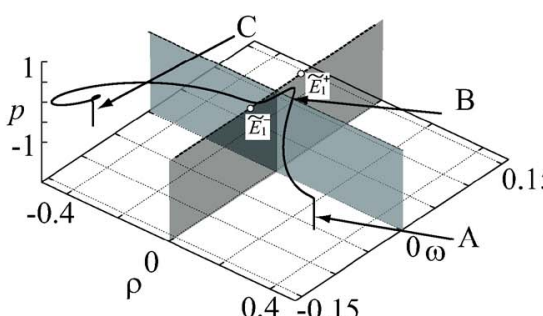

(b)

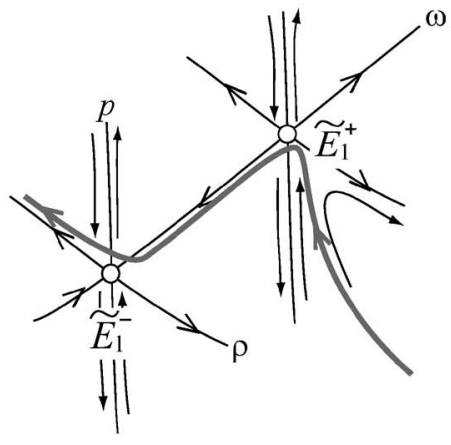

REB

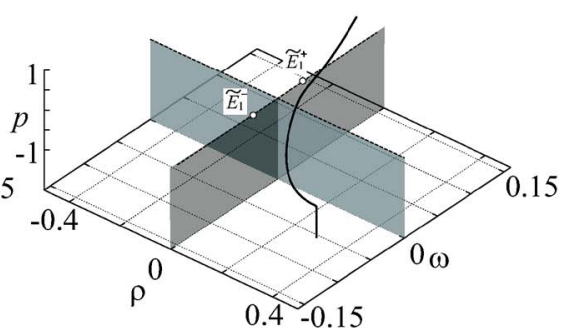

(c) (d)

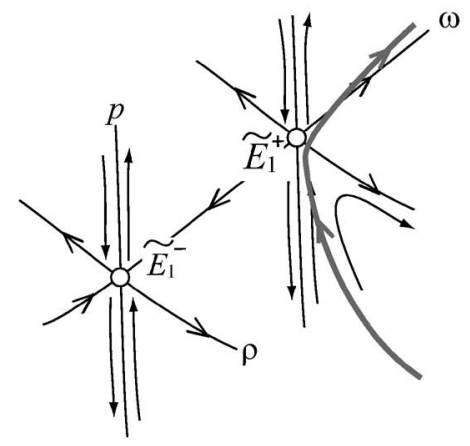

SPL

FIG. 19. (Color online) (a) Penetration: $\left(\mu_{2}, \epsilon\right)=\left(9.3 \times 10^{-3}, 5.7404491 \times 10^{-5}\right)$; (b) rebound: $\left(\mu_{2}, \epsilon\right)=\left(9.3 \times 10^{-3}, 5.7404492 \times 10^{-5}\right)$. Initially, the orbit moves at almost constant velocity (A). Then it becomes closer to the heteroclinic orbit connecting $\widetilde{E}_{1}^{+}$and $\widetilde{E}_{1}^{-}$as parameters are taken closer to TJ (B). Finally, $(\rho, \omega)$ approach $E_{2}^{+}$for (a) and $E_{2}^{-}$for (b), respectively (C). (c) Splitting: $\left(\mu_{2}, \epsilon\right)=\left(9.5 \times 10^{-3}, 5.8062 \times 10^{-5}\right)$. Splitting occurs when the orbit becomes close to the unstable manifold of the upper side emanating from $\widetilde{E}_{1}^{+}$. Computation is stopped when splitting occurs. (d) The schematic figures of flows and orbits. Three types of outputs_-penetration (PEN), rebound (REB), and splitting (SPL) — are observed near TJ.

existence of the heteroclinic connection from $\widetilde{E}_{1}^{+}$to $\widetilde{E}_{1}^{-}$, which plays a key role in understanding the transitions.

First, we consider the transition from penetration to rebound: we take $\mu_{2}$ sufficiently close to TJ with $\mu_{2}<\mu_{\mathrm{TJ}}$, and vary $\epsilon$ for a fixed $\mu_{2}$. As $\epsilon$ is increased from 0 , it is observed that the orbit approaches $\widetilde{E}_{1}^{+}$from the lower side of the $\omega$ $=\sqrt{-\mu_{2}-\epsilon \Gamma_{2}(0)}$ plane [Figs. 19(a) and 19(b)]. As mentioned in the preceding subsection, the lower side of unstable manifold is directed to $\widetilde{E}_{1}^{-}$. Therefore, there exists a critical parameter in between penetration and rebound, at which the orbit converges to $\widetilde{E}_{1}^{-}$. As $\epsilon$ becomes smaller or larger than $\epsilon_{\mathrm{TJ}}$ near the critical point, it is observed that the orbit moves along the unstable manifold of $\widetilde{E}_{1}^{-}$to the right or left side of the $\rho=0$ plane, respectively, followed by the convergence to $E_{2}^{+}$(penetration) or $E_{2}^{-}$(rebound) [see Figs. 19(a) and 19(b)]. Therefore for $\mu_{2}<\mu_{\mathrm{TJ}}$, the fate of the orbit is determined by two consecutive heteroclinic connections; from $E_{2}^{+}$to $\widetilde{E}_{1}^{+}$and then from $\widetilde{E}_{1}^{+}$to $\widetilde{E}_{1}^{-}$.

Second, we consider the case $\mu_{2}>\mu_{\mathrm{TJ}}$ and vary $\epsilon$ as a control parameter for each fixed $\mu_{2}$. From continuity of so- lutions, penetration and rebound occur for small and large $\epsilon$, respectively, and there exists a critical parameter where the orbit touches the $\omega$ axis. In this case, we observe that the orbit passes through the upper side of the $\omega=\sqrt{-\mu_{2}-\epsilon \Gamma_{2}(0)}$ plane [Fig. 19(c)]. Since the unstable manifold of the upper side is directed to $\omega=+\infty, \omega$ diverges to $+\infty$ between the penetration and the rebound regions. Therefore, there exists a splitting regime between penetration and rebound. Summarizing the above discussions, we found two HIOPs $\widetilde{E}_{1}^{ \pm}$and two heteroclinic orbits connecting from $E_{2}^{+}$to $\widetilde{E}_{1}^{+}$and from $\widetilde{E}_{1}^{+}$to $\widetilde{E}_{1}^{-}$. Generally, it should be detected that the orbit crosses the stable manifold by two-parameter searching, since $\widetilde{E}_{1}^{+}$has a one-dimensional stable manifold and a twodimensional unstable one. Moreover, the heteroclinic orbit between $\widetilde{E}_{1}^{+}$to $\widetilde{E}_{1}^{-}$always exists before the saddle-node bifurcation. Therefore, two consecutive heteroclinic connections are formed at the critical parameter where the orbit touches the stable manifold of $\widetilde{E}_{1}^{+}$. Furthermore, TJ is characterized by such a critical parameter since three types of outputspenetration, rebound, and splitting — are observed by unfold- 

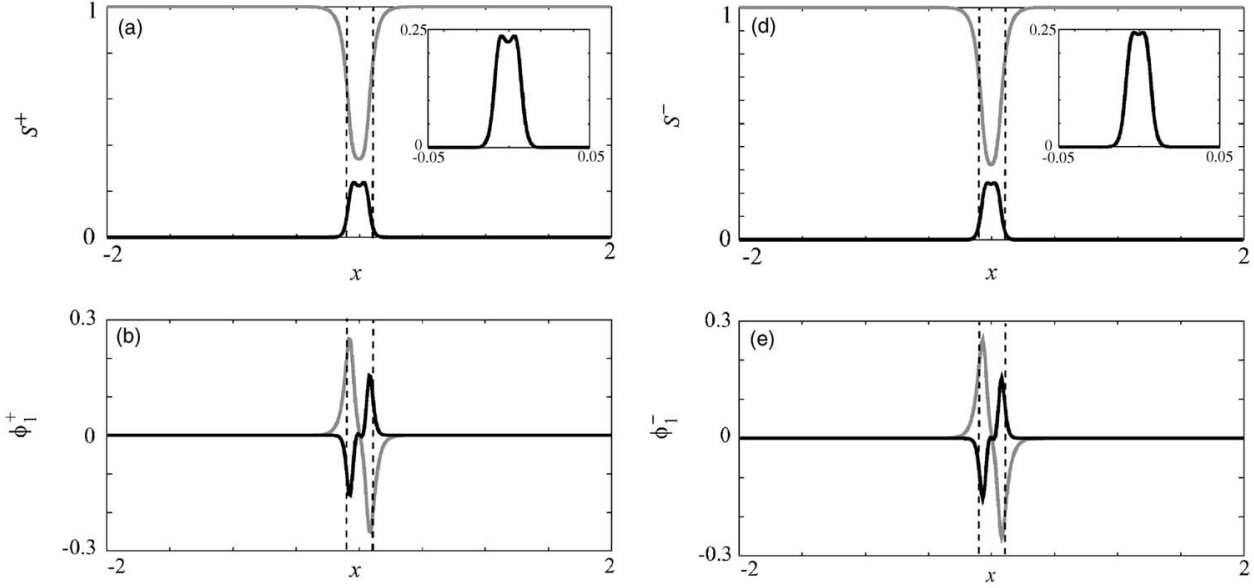

FIG. 20. (a) Profile of $S^{+} . S^{+}$has two positive eigenvalues $\lambda_{1}^{+}=6.724373$ $\times 10^{-3}$ and $\lambda_{2}^{+}=3.655329 \times 10^{-3}$. (b) [(c)] Profile of eigenfunction $\phi_{1}^{+}\left(\phi_{2}^{+}\right)$corresponding to $\lambda_{1}^{+}\left(\lambda_{2}^{+}\right)$. (d) Profile of $S^{-} . S^{-}$has one positive eigenvalues $\quad \lambda_{1}^{-}=3.263519 \times 10^{-3}$. (e) Profile of eigenfunction $\phi_{1}^{-}$corresponding to $\lambda_{1}^{-}$. The solid (gray) lines indicate the $v$ component ( $u$ component). Insets are magnified figures of the central part of the $v$ component of $S^{ \pm}$.

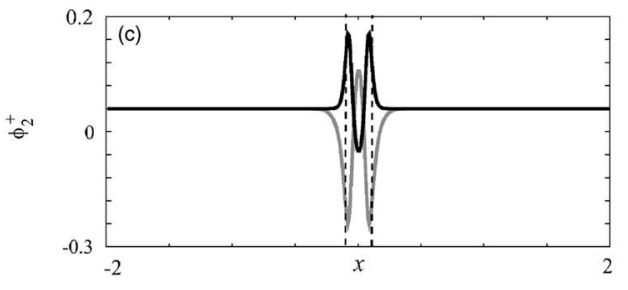

ing the concatenation of two heteroclinic orbits at TJ. In other words, the orbit approaches these two HIOPs and then it is sorted out by the unstable directions emanating from them according to parameters as shown in [Fig. 19(d)].

\section{Comparison between PDE and ODE}

Guided by the analysis of ODEs, we can find two types of HIOPs $S^{ \pm}=S^{ \pm}\left(f_{1}, \epsilon\right)$ corresponding to $\widetilde{E}_{1}^{ \pm}$and two heteroclinic orbits from STP to $S^{+}$and from $S^{+}$to $S^{-}$by taking $\left(f_{1}, \epsilon\right)$ near TJ of Fig. 14. Figure 20 shows the profiles of $S^{ \pm}$ and their unstable eigenfunctions. When we take $f_{1}$ sufficiently close to TJ at the right side of it (Fig. 14), the orbit first approaches $S^{+}$[Fig. 20(a)], then moves to the unstable stationary solution $S^{-}=S^{-}\left(f_{1}, \epsilon\right)$ [Fig. 20(d)]. The orbit becomes very close to the heteroclinic orbit from $S^{+}$to $S^{-}$when parameters are sufficiently close to TJ. As is expected from ODE analysis, when $\left(f_{1}, \epsilon\right)$ is varied near TJ from the opposite side of it, splitting occurs after passing by $S^{+}$.

In order to confirm the fact that the outputs in the PDE system are characterized by the behaviors of unstable manifolds of $S^{ \pm}$, we numerically check the destinations of those unstable manifolds. To do that, we add a small perturbation to $S^{ \pm}$in the direction of the unstable eigenfunctions $\phi_{j}^{ \pm}$as shown in Fig. 20. Note that $S^{+}\left(S^{-}\right)$has two (one) positive eigenvalues. The results are shown in Fig. 21. For positive perturbation of $\phi_{2}^{+}$to $S^{+}$, splitting is observed [Fig. 21(b)]. For negative perturbation of $\phi_{2}^{+}$to $S^{+}$, the transition from $S^{+}$ to $S^{-}$is observed in Fig. 21(c), although it is a bit hard to see in this figure. The behavior is consistent with that of the lower and upper side of the unstable manifold of $\widetilde{E}_{1}^{+}$in the preceding section. We note that the heteroclinic connection between $S^{+}$and $S^{-}$exists for any $f_{1}$ and $\epsilon$, since it originates in the connection of two branches via saddle-node bifurcation [Fig. 21(a)]. For small perturbation of $\phi_{1}^{ \pm}$to $S^{ \pm}$, traveling pulses are observed [Figs. $21(\mathrm{~d})-21(\mathrm{~g})]$. The propagating di- rection is determined by the sign of perturbations, which is consistent with the destinations of the unstable manifold of $\widetilde{E}_{1}^{ \pm}$of the left and right side.

We have confirmed numerically the existence of two HIOPs and the connecting orbits in PDE dynamics near TJ, which is perfectly consistent with the reduced ODEs.

\section{E. Pulse dynamics in periodic media}

The reduced finite-dimensional system (10) remains valid for much more general heterogeneities including periodic media. The system (10) consists of two parts: one is a kind of normal form part of codim 2 singularity and the other is a $\Gamma_{i}$ part. Only the latter part depends on the heterogeneity. In this section, we consider the following spatially periodic type of heterogeneity:

$$
\chi(x)=\sin (2 \pi \Omega x), \quad \Omega>0,
$$

and study the $\Omega$ dependency of pulse dynamics. Moreover, in addition to pinning and penetration, we show by numerics that both PDE and associated ODEs display spatio-temporal chaos under appropriate conditions.

Before discussing complex pulse dynamics, we consider the two extreme cases: slowly varying and highly oscillating media, which correspond to $\Omega \approx 0$ and $\Omega \approx \infty$, respectively. In terms of the ratio $r$ between the size of the pulse and the period of heterogeneity, the former (latter) corresponds to $r$ $=0(r=\infty)$. Also note that the slowly varying case is related to the following question: when the height $\epsilon$ is high enough so that the pulse bounces off at the bump, then is it possible for the pulse to go across it when the slope $\gamma$ becomes less steep? The answer is given by one of the consequences of the following proposition.

Proposition 1. (a) $\Omega \rightarrow 0$ (slowly varying case), then $\Gamma_{1}(p)$ and $\dot{\Gamma}_{j}(p)(\cdot=d / d p, j=1,2) \rightarrow 0$ uniformly with respect 


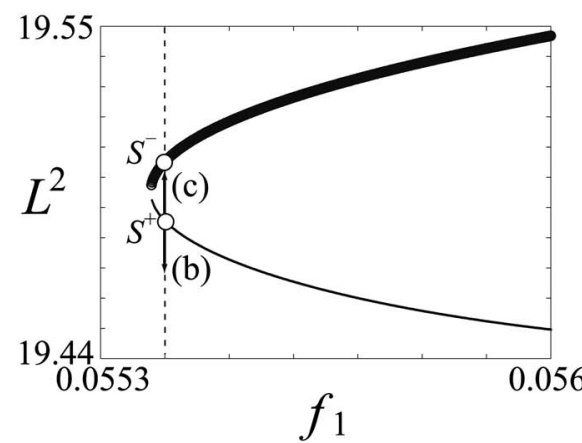

(a)

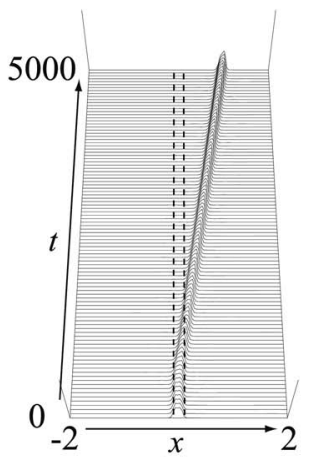

(d)

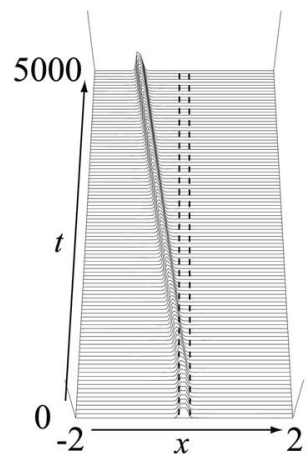

(e)

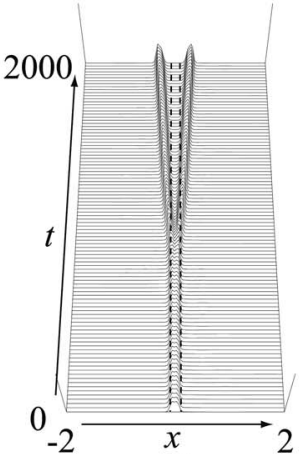

(b)

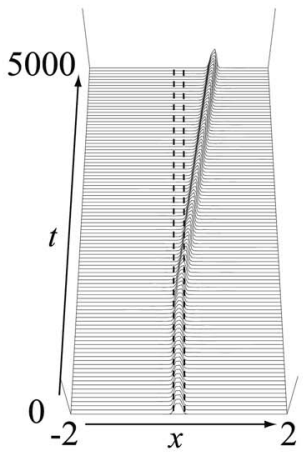

(f)

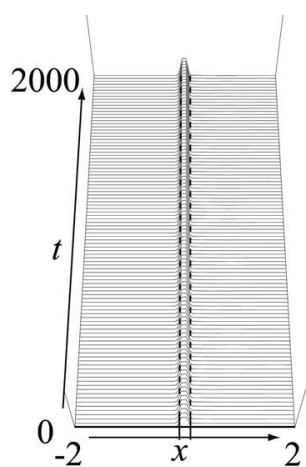

(c)

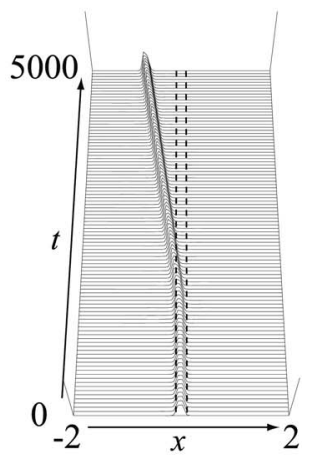

(g)
FIG. 21. (a) Bifurcation diagram for $S^{ \pm}$for $\epsilon=2.14 \times 10^{-4}$. The bold line indicates the unstable stationary solution, which has a drift instability. The thin line indicates the unstable stationary solution, which has drift and splitting instability. (b) [(c)] Outputs of small positive (negative) perturbation of $\phi_{2}^{+}$to $S^{+}$. For negative perturbation, the solution approaches $S^{-}$. (d) [(e)] Outputs of small positive (negative) perturbation of $\phi_{1}^{+}$to $S^{+}$. (f) $[(\mathrm{g})]$ Outputs of small positive (negative) perturbation of $\phi_{1}^{-}$to $S^{-}$. to $p$. (b) $\Omega \rightarrow+\infty$ (highly oscillating case), then $\Gamma_{j}(p)(j$ $=1,2) \rightarrow 0$ uniformly with respect to $p$.

Recalling the definition of $\Gamma_{j}$ [see Eq. (A5)] and the odd symmetry of $\phi^{*}$, the first part of Proposition 1 is clear. Since $\sin (2 \pi \Omega x)$ weakly converges to 0 as $\Omega \rightarrow+\infty$, the second part also holds. When the slope becomes less steep (i.e., $\Omega$ $\rightarrow 0$ ) with keeping the same height as before, then the effect coming from the heterogeneity in the second equation of Eq. (10) becomes small from Proposition 1(a) and hence the pulse can transmit below some threshold value of the slope, although the pulse velocity gradually changes depending on its position. On the other hand, for the highly oscillating case, Fig. 22(b) clearly shows that the pulse does not feel any barrier of heterogeneity as is expected from Proposition 1(b), even though it cannot penetrate it for moderate frequency as in Fig. 22(a). The above discussion suggests that the period of the media should be comparable to the size of the traveling pulse in order to observe an exotic dynamics.

Recalling the discussions in the preceding sections, separators of standing pulses are also distributed periodically and the pulse moves around among those saddles. One may expect, therefore, the occurrence of complex behaviors of pulses under such environments. In fact, we find a chaotic pulse dynamics (CPD) of Eq. (9) near the codim2 (DR + SN) point with $\Omega=3$ as in Fig. 23(a). In addition, executing a three-parameter search with respect to $\mu_{2}, \epsilon$, and $\Omega$ for Eq. (10), we find a similar CPD as in Figs. 23(b) and 23(c). In both PDE and ODEs simulations, CPD are detected near TJ around which penetration, rebound, and splitting are observed. As discussed in the preceding sections, the solution orbit becomes closer to the heteroclinic orbit connecting two unstable solutions as shown in Figs. 14 and 19 when the associated parameters are close to $\mathrm{TJ}$, which suggests that the emergence of the heteroclinic orbit is responsible for CPD. Summarizing the above discussions, we numerically find the following properties for the onset of CPD:

(a) CPD appears when the period of heterogeneity and the size of the pulse are comparable.

(b) CPD appears near the triple junction point where penetration, rebound, and splitting converge.

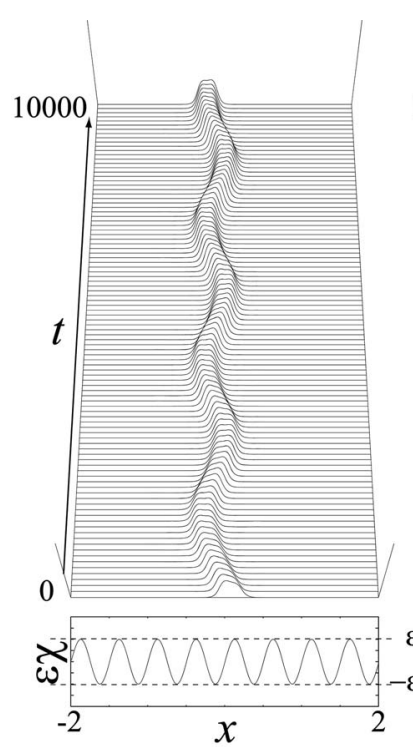

(a)

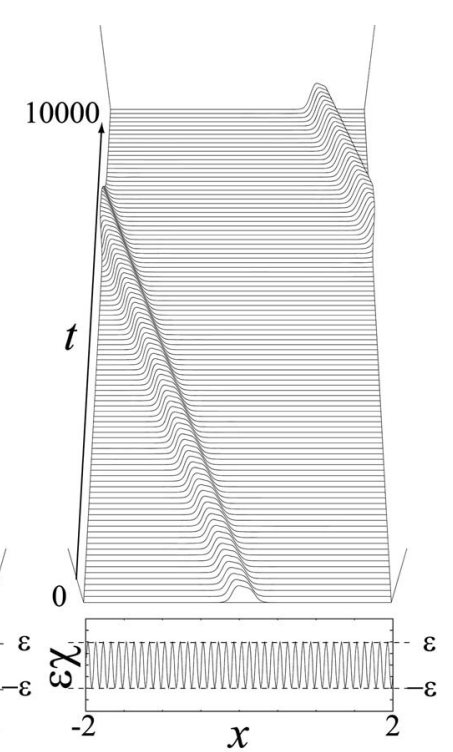

(b)
FIG. 22. Pulse dynamics in periodic media for $\epsilon=0.01$. (a) The pulse cannot penetrate the barrier and a zigzag motion is observed when the period of heterogeneity is comparable compared with the size of the pulse. (b) The effect of heterogeneity becomes negligible for highly oscillating media (i.e., $\Omega$ tends to $\infty$ ). 


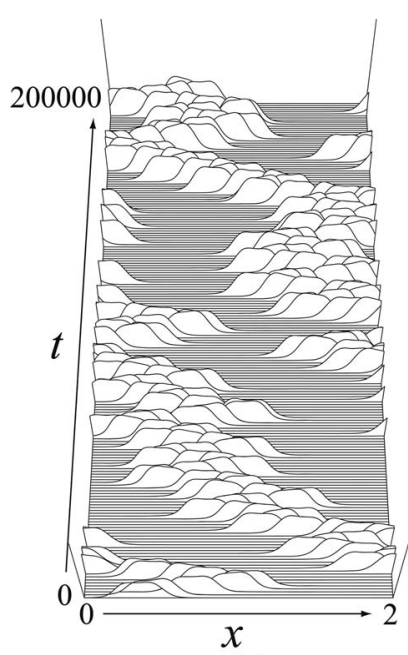

(a)

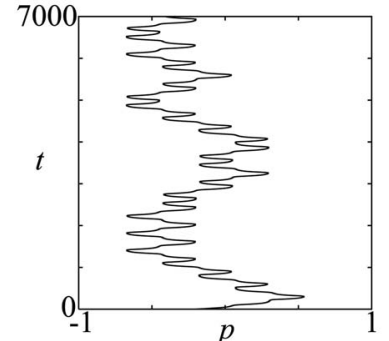

(b)

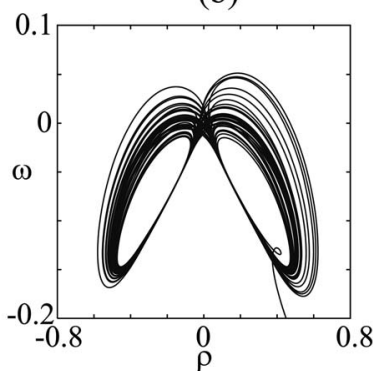

(c)
FIG. 23. (a) Spatio-temporal chaos for PDE (9) with $f_{0}=0.024, f_{1}$ $=0.0555535, \tau=1.007734$, and $\epsilon=1.035 \times 10^{-4}, \Omega=3$. (b) and (c) Chaotic dynamics of ODE (10) with (12) for $\left(\mu_{2}, \epsilon, \Omega\right)=\left(1.0 \times 10^{-3}, 2.1 \times 10^{-4}, 4\right)$.

Note that pulse dynamics with periodic heterogeneity is also studied in Ref. 44 for the Gray-Scott model in which the heterogeneity is introduced in the diffusivity and CPD is detected near a codim 2 singularity of drift and Hopf instabilities. A complete understanding of CPD near the codim 2 point remains a challenge for the future.

\section{CONCLUDING REMARKS}

We studied the dynamic behaviors of traveling pulses in heterogeneous media of bump and periodic types. A variety of responses are observed when they encounter heterogeneity such as: rebound, oscillation, pinning to a steady or oscillatory state, splitting, and penetration. This originates from a combination of hidden instabilities embedded in pulses and external perturbations from the heterogeneity. Here hidden means that the traveling pulses are asymptotically stable for our experiments, however the associated parameter value is close to a singularity of codim 1 or 2 in the parameter space. The model systems employed here are the three-component system of Eq. (1) and the Gray-Scott model (9). When we introduced heterogeneities to those models, many defects, i.e., new standing pulses created near the edge of the bump, appear for Eq. (1), and they play a key role to classify the various responses in the phase diagram of Figs. 1(c) and 6(a). Pinning-depinning transition, for instance, can be explained by heteroclinic and homoclinic bifurcations among those defects. On the other hand, the Gray-Scott model does not have such defects and keeps the trivial constant state $(1,0)$ as its background state even for the heterogeneous case. This is because the heterogeneity of Eq. (9) appears in a multiplicative way, not in an additive way like Eq. (1). Nevertheless, Eq. (9) has two different types of steady states as shown in Fig. 20 that originate from two types of unstable standing pulses for the homogeneous case, in fact they converge to the corresponding standing pulses of the homogeneous system as the width $d$ of the bump goes to $\infty$. The above defects and the standing pulses created by heterogeneity are part of HIOPs (heterogeneity-induced ordered patterns), which play a key role for understanding the pulse dynamics in heterogeneous media. It should be remarked that all the characteristic features of pulse dynamics in heterogeneous media can be found in the reduced finite-dimensional systems like Eqs. (8) and (10), which actually not only clarifies the existence and stabilities of HIOPs, but also shows us global interrelations among them that is crucial for understanding the transitions of pulse responses. Although we showed a complex dynamics for periodic media, such complexity is already implicitly suggested in the analysis for the bump case like the heteroclinic connection among different HIOPs. More detailed analysis is yet to be done in this direction.

\section{ACKNOWLEDGMENTS}

The authors would like to thank Markus Bär and the anonymous referees for constructive comments. This work was partially supported by the Grant-in-Aid for Scientific Research (A)16204008 and the Ministry of Education, Science, Sports and Culture of Japan, Grant-in-Aid for Young Scientists (B) 18740050 and (B) 18740238 .

\section{APPENDIX: REDUCTION TO FINITE-DIMENSIONAL DYNAMICS FOR CODIM2 SINGULARITY}

Let us derive a finite-dimensional ODE near the codim2 point for the following general 1D reaction-diffusion system:

$$
\boldsymbol{u}_{t}=D \boldsymbol{u}_{x x}+F(\boldsymbol{u} ; k)=: \mathcal{A}(\boldsymbol{u} ; \boldsymbol{k}), \quad t>0, x \in \mathbb{R},
$$

where $\boldsymbol{k}=\left(k_{1}, k_{2}\right) \in \mathbb{R}^{2}$. We assume the following.

(S1') There exist $\boldsymbol{k}=\boldsymbol{k}^{c}=\left(k_{1}^{c}, k_{2}^{c}\right)$ such that the nontrivial spatially symmetric standing pulse solution $\boldsymbol{u}=S(x ; \boldsymbol{k})$ of Eq. (A1) exists.

We consider Eq. (A1) in a neighborhood of $\boldsymbol{k}=\boldsymbol{k}^{c}$, with small parameters $\boldsymbol{\eta}=\left(\eta_{1}, \eta_{2}\right)$ as $k_{1}=k_{1}^{c}+\eta_{1}$ and $k_{2}=k_{2}^{c}+\eta_{2}$. We assume that Eq. (A1) takes the following form:

$$
\boldsymbol{u}_{t}=\mathcal{A}(\boldsymbol{u} ; \boldsymbol{k})+\left[\eta_{1}+\epsilon \chi(x)\right] \boldsymbol{g}_{1}(\boldsymbol{u})+\eta_{2} \boldsymbol{g}_{2}(\boldsymbol{u}),
$$

where $\boldsymbol{g}_{1}$ and $\boldsymbol{g}_{2}$ are $N$-dimensional vector-valued functions.

Let $L:=\mathcal{A}^{\prime}\left(S(x) ; \boldsymbol{k}^{c}\right)$. We assume the following:

(S2') The spectral set of $L$ consists of two sets $\sigma=\{0\}$ and $\sigma_{2} \subset\left\{\mu \in \mathrm{C} ; \operatorname{Re}(\mu)<-\gamma_{0}\right\}$, where $\gamma_{0}$ is a positive constant.

$\left(\mathrm{S}^{\prime}\right) L$ has a codim 2 singularity at $\eta_{1}=\eta_{2}=0$ consisting of drift and saddle-node bifurcations besides the translationfree 0 eigenvalue. That is, there exist three eigenfunctions $\phi(x), \psi(x)$, and $\xi(x)$ such that

$$
L \phi=0, \quad L \psi=-\phi, \quad L \xi=0,
$$

where $\phi=\partial S / \partial x$. Note that $\phi(x)$ and $\psi(x)$ are odd functions, and $\xi(x)$ is an even function. $\psi(x)$ represents the deformation vector with Jordan form for the drift bifurcation, and $\xi(x)$ is the eigenfunction for the saddle-node bifurcation.

Similar properties also hold for $L^{*}$. That is, there exist $\phi^{*}, \xi^{*}$, and $\psi^{*}$ such that $L^{*} \phi^{*}=L^{*} \xi^{*}=0$ and $L^{*} \psi^{*}=-\phi^{*}$, 
where $\phi^{*}(x)$ and $\psi^{*}(x)$ are odd functions and $\xi^{*}(x)$ is an even function. Let $\phi^{*}, \psi, \psi^{*}, \xi$, and $\xi^{*}$ be normalized by

$$
\langle\psi, \phi\rangle=\left\langle\psi, \psi^{*}\right\rangle=0, \quad\left\langle\xi, \xi^{*}\right\rangle=\left\langle\phi, \psi^{*}\right\rangle=1 .
$$

$\left(\mathrm{S} 4^{\prime}\right)$ Each element of eigenfunctions $\phi, \phi^{*}, \psi, \psi^{*}, \xi$, and $\xi^{*}$ decays exponentially as $|x| \rightarrow+\infty$.

The above assumptions are numerically confirmed for Eq. (9). The eigenfunctions are depicted in Fig. 15.

Our ansatz is the following:

$\boldsymbol{u}=S(x-p)+q \psi(x-p)+r \xi(x-p)+\zeta^{\dagger}(x-p)+\boldsymbol{w}$,

where $p, q$, and $r$ are scalar functions of $t ; \zeta^{\dagger} \in \tilde{E}$ : $=\operatorname{span}\{\phi, \psi, \xi\}$. The remaining two terms $\zeta^{\dagger}$ and $\boldsymbol{w}$ belong to $\widetilde{E}^{\perp}$. More precisely, $\zeta^{\dagger}=q^{2} \zeta_{1}+r^{2} \zeta_{2}+q r \zeta_{3}+\eta_{1} \zeta_{4}+\eta_{2} \zeta_{5}$ with $\zeta_{j} \in E^{\perp}$ being determined by

$L \zeta_{1}+\frac{1}{2} F^{\prime \prime}(S) \psi^{2}+\psi_{x}=\alpha_{1} \xi, \quad L \zeta_{2}+\frac{1}{2} F^{\prime \prime}(S) \xi^{2}=\alpha_{2} \xi$,

$L \zeta_{3}+F^{\prime \prime}(S) \psi \cdot \xi+\xi_{x}=\alpha_{3} \psi+\alpha_{3}^{\prime} \phi$

$L \zeta_{4}+g_{1}(S)=\alpha_{4} \xi, \quad L \zeta_{5}+g_{2}(S)=\alpha_{5} \xi$

where $\alpha_{j}$ and $\alpha_{j}^{\prime}$ are constants satisfying the following equations:

$$
\begin{aligned}
& \left\langle\frac{1}{2} F^{\prime \prime}(S) \psi^{2}+\psi_{x}-\alpha_{1} \xi, \xi^{*}\right\rangle=0, \\
& \left\langle\frac{1}{2} F^{\prime \prime}(S) \xi^{2}-\alpha_{2} \xi, \xi^{*}\right\rangle=0, \\
& \left\langle F^{\prime \prime}(S) \psi \cdot \xi+\xi_{x}-\alpha_{3} \psi, \phi^{*}\right\rangle=0, \\
& \left\langle F^{\prime \prime}(S) \psi \cdot \xi+\xi_{x}-\alpha_{3} \phi, \psi^{*}\right\rangle=0, \\
& \left\langle\boldsymbol{g}_{1}(S)-\alpha_{4} \xi, \xi^{*}\right\rangle=0, \\
& \left\langle\boldsymbol{g}_{2}(S)-\alpha_{5} \xi, \xi^{*}\right\rangle=0 .
\end{aligned}
$$

By substituting Eq. (A3) into Eq. (A2) and taking inner products of Eq. (A2) with $\phi^{*}, \psi^{*}$, and $\xi^{*}$, we have

$$
\begin{aligned}
\dot{p}= & q-\alpha_{3}^{\prime} q r-\epsilon \Gamma_{0}(p) \\
& +O\left[|q|^{3}+|r|^{3}+|\boldsymbol{\eta}|_{2}^{3 / 2}+|\epsilon|\left(|q|+|r|+|\boldsymbol{\eta}|_{2}+|\epsilon|\right)\right], \\
\dot{q}= & \left(M_{30} q^{2}+M_{11} r+M_{12} r^{2}+\mu_{1}\right) q+\epsilon \Gamma_{1}(p) \\
& +O\left[\left(|q|+|r|+|\boldsymbol{\eta}|_{2}\right)\left(|q|^{3}+|r|^{3}+|\boldsymbol{\eta}|_{2}^{3 / 2}\right)\right. \\
& \left.+|\epsilon|\left(|q|+|r|+|\boldsymbol{\eta}|_{2}+|\epsilon|\right)\right], \\
\dot{r}= & N_{20} q^{2}+N_{02} r^{2}+N_{21} q^{2} r+N_{03} r^{3}+\mu_{3} r+\mu_{2}+\epsilon \Gamma_{2}(p) \\
& +O\left[\left(|q|+|r|+|\boldsymbol{\eta}|_{2}\right)\left(|q|^{3}+|r|^{3}+|\boldsymbol{\eta}|_{2}^{3 / 2}\right)\right. \\
& \left.+|\epsilon|\left(|q|+|r|+|\boldsymbol{\eta}|_{2}+|\epsilon|\right)\right],
\end{aligned}
$$

where $|\boldsymbol{\eta}|_{2}:=\sqrt{\eta_{1}^{2}+\eta_{2}^{2}}$ and

$$
\begin{aligned}
& \Gamma_{0}(p)=\int_{-\infty}^{\infty} \chi(x) \boldsymbol{g}_{1}[S(x-p)] \cdot \psi^{*}(x-p) d x, \\
& \Gamma_{1}(p)=\int_{-\infty}^{\infty} \chi(x) \boldsymbol{g}_{1}[S(x-p)] \cdot \phi^{*}(x-p) d x,
\end{aligned}
$$

$\Gamma_{2}(p)=\int_{-\infty}^{\infty} \chi(x) g_{1}[S(x-p)] \cdot \xi^{*}(x-p) d x$,

$M_{11}=\alpha_{3}$,

$M_{30}=\left\langle F^{\prime \prime}(S) \psi \cdot \zeta_{1}, \phi^{*}\right\rangle+\frac{1}{6}\left\langle F^{\prime \prime \prime}(S) \psi^{3}, \phi^{*}\right\rangle+\left\langle\partial_{x} \zeta_{1}, \phi^{*}\right\rangle$,

$M_{12}=\left\langle F^{\prime \prime}(S) \xi \cdot \zeta_{3}, \phi^{*}\right\rangle+\left\langle F^{\prime \prime}(S) \psi \cdot \zeta_{2}, \phi^{*}\right\rangle$ $+\frac{1}{2}\left\langle F^{\prime \prime \prime}(S) \psi \cdot \xi^{2}, \phi^{*}\right\rangle+\left\langle\partial_{x} \zeta_{2}, \phi^{*}\right\rangle-\alpha^{\prime}{ }_{3}\left\langle\xi_{x}, \phi^{*}\right\rangle$,

$N_{20}=\alpha_{1}, \quad N_{02}=\alpha_{2}$,

$N_{21}=\left\langle F^{\prime \prime}(S) \xi \cdot \zeta_{1}, \xi^{*}\right\rangle+\left\langle F^{\prime \prime}(S) \psi \cdot \zeta_{3}, \xi^{*}\right\rangle$ $+\frac{1}{2}\left\langle F^{\prime \prime \prime}(S) \psi^{2} \cdot \xi, \xi^{*}\right\rangle+\left\langle\partial_{x} \zeta_{3}, \xi^{*}\right\rangle-\alpha_{3}^{\prime}\left\langle\psi_{x}, \xi^{*}\right\rangle$,

$N_{03}=\left\langle F^{\prime \prime}(S) \xi \cdot \zeta_{2}, \xi^{*}\right\rangle+\frac{1}{6}\left\langle F^{\prime \prime \prime}(S) \xi^{3}, \xi^{*}\right\rangle$,

$\mu_{1}=a_{1} \eta_{1}+a_{2} \eta_{2}$,

$\mu_{2}=\alpha_{4} \eta_{1}+\alpha_{5} \eta_{2}+c_{1} \eta_{1}^{2}+c_{2} \eta_{1} \eta_{2}+c_{3} \eta_{2}^{2}$

$\mu_{3}=b_{1} \eta_{1}+b_{2} \eta_{2}$,

$a_{1}=\left\langle\boldsymbol{g}_{1}^{\prime}(S) \psi, \phi^{*}\right\rangle+\left\langle F^{\prime \prime}(S) \psi \cdot \zeta_{4}, \phi^{*}\right\rangle$,

$a_{2}=\left\langle\mathbf{g}_{2}^{\prime}(S) \psi, \phi^{*}\right\rangle+\left\langle F^{\prime \prime}(S) \psi \cdot \zeta_{5}, \phi^{*}\right\rangle$

$b_{1}=\left\langle\mathbf{g}_{1}^{\prime}(S) \xi, \xi^{*}\right\rangle+\left\langle F^{\prime \prime}(S) \xi \cdot \zeta_{4}, \xi^{*}\right\rangle$,

$b_{2}=\left\langle\boldsymbol{g}_{2}^{\prime}(S) \xi, \xi^{*}\right\rangle+\left\langle F^{\prime \prime}(S) \xi \cdot \zeta_{5}, \xi^{*}\right\rangle$,

$c_{1}=\frac{1}{2}\left\langle F^{\prime \prime}(S) \zeta_{4}^{2}, \xi^{*}\right\rangle, \quad c_{2}=\left\langle F^{\prime \prime}(S) \zeta_{4} \cdot \zeta_{5}, \xi^{*}\right\rangle$,

$c_{3}=\frac{1}{2}\left\langle F^{\prime \prime}(S) \zeta_{5}^{2}, \xi^{*}\right\rangle$.

Using the following notation,

$\widetilde{q}=q+K q r, \quad \widetilde{r}=r+e_{3} r^{2}, \quad d t=\left(1+e_{1} r\right) d \tau$,

$\mu^{\prime}{ }_{1}=\mu_{1}+K \mu_{2}, \quad \mu_{2}^{\prime}=\mu_{2}, \quad \mu^{\prime}{ }_{3}=\mu_{3}$,

where $K=-M_{30} / N_{20}, e_{1}=-N_{03} / N_{02}, e_{3}=K-e_{1} / 2-N_{21} / 2 N_{20}$, and

$$
\begin{aligned}
& C_{0}=M_{11}, \\
& C_{1}=M_{12}-\frac{N_{02} M_{30}}{N_{20}}-M_{11}\left(\frac{3 N_{03}}{2 N_{02}}-\frac{M_{30}}{N_{20}}-\frac{N_{21}}{2 N_{20}}\right), \\
& C_{2}=N_{02}, \quad C_{3}=N_{20},
\end{aligned}
$$

we rewrite Eq. (A4) in the following form:

$$
\begin{aligned}
\dot{p}= & \widetilde{q}-\left(K+e_{1}+\alpha_{3}^{\prime}\right) \widetilde{q} \widetilde{r}-\epsilon \Gamma_{0}(p) \\
& +O\left[|\widetilde{q}|^{3}+|\widetilde{r}|^{3}+|\boldsymbol{\eta}|_{2}^{3 / 2}+|\epsilon|\left(|\widetilde{q}|+|\widetilde{r}|+|\boldsymbol{\eta}|_{2}+|\epsilon|\right)\right],
\end{aligned}
$$




$$
\begin{aligned}
\dot{\tilde{q}}= & \mu_{1}^{\prime} \widetilde{q}+C_{0} \widetilde{q} \widetilde{r}+C_{1} \widetilde{q} \widetilde{r}^{2}+\epsilon \Gamma_{1}(p) \\
& +O\left[\left(|\widetilde{q}|+|\widetilde{r}|+|\boldsymbol{\eta}|_{2}\right)\left(|\widetilde{q}|^{3}+|\widetilde{r}|^{3}+|\boldsymbol{\eta}|_{2}^{3 / 2}\right)\right. \\
& \left.+|\epsilon|\left(|\widetilde{q}|+|\widetilde{r}|+|\boldsymbol{\eta}|_{2}+|\epsilon|\right)\right], \\
\dot{\widetilde{r}}= & \mu_{2}^{\prime}+C_{2} \widetilde{r}^{2}+C_{3} \widetilde{q}^{2}+\mu_{3}^{\prime} \tilde{r}+\epsilon \Gamma_{2}(p) \\
& +O\left[\left(|\widetilde{q}|+|\widetilde{r}|+|\boldsymbol{\eta}|_{2}\right)\left(|\widetilde{q}|^{3}+|\widetilde{r}|^{3}+|\boldsymbol{\eta}|_{2}^{3 / 2}\right)\right. \\
& \left.+|\epsilon|\left(|\widetilde{q}|+|\widetilde{r}|+|\boldsymbol{\eta}|_{2}+|\epsilon|\right)\right] .
\end{aligned}
$$

Replacing

$$
\begin{aligned}
& \rho=\frac{\left|C_{1}\right|}{C_{2}} \widetilde{q}, \quad \omega=\frac{\left|C_{1}\right|}{C_{2}}\left(\tilde{r}+\frac{\mu_{3}^{\prime}}{2 C_{2}}\right), \quad \tau=\frac{C_{2}^{2}}{\left|C_{1}\right|} t, \\
& \tilde{\mu}_{1}=\frac{C_{2}^{2}}{\left|C_{1}\right|} \mu_{1}^{\prime}, \quad \tilde{\mu}_{2}=\frac{C_{2}^{3}}{C_{1}^{2}} \mu_{2}^{\prime}, \\
& \theta_{1}=\frac{C_{0}}{C_{2}}, \quad \theta_{2}=-\frac{C_{3}}{C_{2}}, \quad \tilde{\epsilon}=\frac{C_{1}^{2}}{C_{2}^{3}} \epsilon,
\end{aligned}
$$

and omitting the tildes for simplicity, we finally reach the following form:

$$
\begin{aligned}
\dot{p}= & \frac{1}{C_{2}} \rho-\frac{K+e_{1}+\alpha_{3}^{\prime}}{\left|C_{1}\right|} \rho \omega-\frac{\left|C_{1}\right|}{C_{2}^{2}} \epsilon \Gamma_{0}(p) \\
& +O\left[|\rho|^{3}+|\omega|^{3}+|\boldsymbol{\eta}|_{2}^{3 / 2}+|\epsilon|\left(|\rho|+|\omega|+|\boldsymbol{\eta}|_{2}+|\epsilon|\right)\right], \\
\dot{\rho}= & \rho\left(s \mu_{1}+\theta_{1} \omega\right)+\epsilon \Gamma_{1}(p) \\
& +O\left[\left|\rho \omega^{2}\right|+\left(|\rho|+|\omega|+|\boldsymbol{\eta}|_{2}\right)\left(|\rho|^{3}+|\omega|^{3}+|\boldsymbol{\eta}|_{2}^{3 / 2}\right)\right. \\
& \left.+|\epsilon|\left(|\rho|+|\omega|+|\boldsymbol{\eta}|_{2}+|\epsilon|\right)\right], \\
\dot{\omega}= & \mu_{2}+\omega^{2}-\theta_{2} \rho^{2}+\epsilon \Gamma_{2}(p) \\
& +O\left[\left(|\rho|+|\omega|+|\boldsymbol{\eta}|_{2}\right)\left(|\rho|^{3}+|\omega|^{3}+|\boldsymbol{\eta}|_{2}^{3 / 2}\right)\right. \\
& \left.+|\epsilon|\left(|\rho|+|\omega|+|\boldsymbol{\eta}|_{2}+|\epsilon|\right)\right],
\end{aligned}
$$

where $s=\operatorname{sgn}\left[C_{1}\right]= \pm 1$.

We fix $\left|\mu_{1}\right|$ to be sufficiently small and take $\mu_{2}$ $=O\left(\left|\mu_{1}\right|^{2-\nu}\right)$ and $\epsilon=O\left(\left|\mu_{1}\right|^{2-\nu}\right)(2 / 3<\nu<1)$. Then, in the first equation of Eqs. (A6), $O(\rho)$ becomes the leading order, and we discard the terms higher than $\rho$. In the second and third equations of Eqs. (A6), we consider the regime where $\rho$ $=O\left(\left|\mu_{1}\right|^{1-\nu}\right)$ and $\omega=O\left(\left|\mu_{1}\right|^{1-\nu}\right)$, and discard the terms of order higher than $\left|\mu_{1}\right|^{2}$. Then the principal part of Eqs. (A6) is

$$
\begin{aligned}
& \dot{p}=\rho / C_{2}, \quad \dot{\rho}=\rho\left(s \mu_{1}+\theta_{1} \omega\right)+\epsilon \Gamma_{1}(p), \\
& \dot{\omega}=\mu_{2}+\omega^{2}-\theta_{2} \rho^{2}+\epsilon \Gamma_{2}(p) .
\end{aligned}
$$

${ }^{1}$ Y. A. Astrov and H.-G. Purwins, Phys. Lett. A 283, 349 (2001). ${ }^{2}$ M. Bär, M. Eiswirth, H.-H. Rotermund, and G. Ertl, Phys. Rev. Lett. 69, 945 (1992).
${ }^{3}$ M. Bode, A. W. Liehr, C. P. Schenk, and H.-G. Purwins, Physica D 161, 45 (2002).

${ }^{4}$ P. de Kepper, J.-J. Perraud, B. Rudovics, and E. Dulos, Int. J. Bifurcation Chaos Appl. Sci. Eng. 4, 1215 (1994).

${ }^{5}$ K. J. Lee, W. D. McCormick, J. E. Pearson, and H. L. Swinney, Nature (London) 369, 215 (1994).

${ }^{6}$ A. von Oertzen, A. S. Mikhailov, H.-H. Rotermund, and G. Ertl, J. Phys. Chem. B 102, 4966 (1998).

${ }^{7}$ C. P. Schenk, M. Or-Guil, M. Bode, and H.-G. Purwins, Phys. Rev. Lett. 78, 3781 (1997).

${ }^{8}$ M. Argentina, P. Coullet, and L. Mahadevan, Phys. Rev. Lett. 79, 2803 (1997).

${ }^{9}$ S.-I. Ei, J. Dyn. Differ. Equ. 14, 85 (2002).

${ }^{10}$ S.-I. Ei, M. Mimura, and M. Nagayama, Physica D 165, 176 (2002).

${ }^{11}$ Y. Hayase and T. Ohta, Phys. Rev. E 62, 5998 (2000).

${ }^{12}$ K. Krischer and A. Mikhailov, Phys. Rev. Lett. 73, 3165 (1994).

${ }^{13}$ J. H. Merkin, V. Petrov, S. K. Scott, and K. Showalter, Phys. Rev. Lett. 76, 546 (1996).

${ }^{14}$ Y. Nishiura, T. Teramoto, and K.-I. Ueda, Phys. Rev. E 67, 056210 (2003).

${ }^{15}$ Y. Nishiura, T. Teramoto, and K.-I. Ueda, Chaos 13, 962 (2003).

${ }^{16}$ Y. Nishiura, Far-from-equilibrium Dynamics (AMS, Metals Park, OH, 2003).

${ }^{17}$ Y. Nishiura and D. Ueyama, Physica D 150, 137 (2001).

${ }^{18}$ T. Ohta, Physica D 151, 61 (2001).

${ }^{19}$ H. G. Purwins, Y. A. Astrov, and I. Brauer, in Proceedings of the Fifth Experimental Chaos Conference, edited by M. Ding, W. L. Ditto, L. M. Pecora, and M. L. Spano (World Scientific, Singapore, 2001), pp. 3-13.

${ }^{20}$ W. N. Reynolds, S. Ponce-Dawson, and J. E. Pearson, Phys. Rev. E 56, 185 (1997).

${ }^{21}$ T. Teramoto, K.-I. Ueda, and Y. Nishiura, Phys. Rev. E 69, 056224 (2004)

${ }^{22}$ M. G. Zimmermann, S. O. Firle, M. A. Natiello, M. Hildebrand, M. Eiswirth, M. Bär, A. K. Bangia, and I. G. Kevrekidis, Physica D 110, 92 (1997).

${ }^{23}$ J. Keener and J. Sneyd, Mathematical Physiology (Springer-Verlag, New York, 1998)

${ }^{24}$ Y. Nishiura and D. Ueyama, Physica D 130, 73 (1999).

${ }^{25}$ B. S. Kerner and V. V. Osipov, Autosolitons: A New Approach to Problems of Self-Organization and Turbulence (Kluwer, Dordrecht, 1994).

${ }^{26}$ Y. Nishiura, T. Teramoto, and K.-I. Ueda, "Collision dynamics of traveling pulses near singularities" (unpublished).

${ }^{27}$ J. Xin, SIAM Rev. 42, 161 (2000).

${ }^{28}$ E. J. Doedel et al., AUTO 2000: Continuation and bifurcation software for ordinary differential equations, http://cmvl.cs.concordia.ca/auto

${ }^{29}$ Y. Nishiura, Y. Oyama, and K.-I. Ueda, Hokkaido Math. J. 36, 207 (2007)

${ }^{30}$ M. Gutman, I. Aviram, and A. Rabinovitch, Phys. Rev. E 69, 016211 (2004)

${ }^{31}$ M. Gutman, I. Aviram, and A. Rabinovitch, Phys. Rev. E 70, 037202 (2004).

${ }^{32}$ J. P. Pauwelussen, J. Math. Biol. 15, 151 (1982).

${ }^{33}$ H. Ikeda and M. Mimura, SIAM J. Appl. Math. 49, 515 (1989).

${ }^{34}$ N. Kinezaki, K. Kawasaki, F. Takasu, and N. Shigesada, Theor. Popul. Biol. 64, 291 (2003).

${ }^{35}$ J. P. Pauwelussen, Physica D 4, 67 (1981).

${ }^{36}$ M. Bode, Physica D 106, 270 (1997).

${ }^{37}$ P. Schutz, M. Bode, and H. G. Purwins, Physica D 82, 382 (1995).

${ }^{38}$ Y.-X. Li, Physica D 186, 27 (2003).

${ }^{39}$ S.-I. Ei, Y. Nishiura, and K.-I. Ueda, Jpn. J. Ind. Appl. Math. 18, 181 (2001).

${ }^{40}$ A. Prat, Y.-X. Li, and P. Bressloff, Physica D 202, 177 (2005).

${ }^{41}$ X. Yuan, T. Teramoto, and Y. Nishiura, Phys. Rev. E 75, 036220 (2007)

${ }^{42}$ K.-I. Ueda, T. Teramoto, and Y. Nishiura, Gakuto International Series: Mathematical Sciences and Applications, Vol. 22, p. 205 (Gakkotosho, Tokyo, 2005).

${ }^{43}$ C. Utzny and M. Bär (unpublished).

${ }^{44}$ D. Ueyama, RIMS Kokyuroku 1453, 18 (2005) (in Japanese). 